\title{
CALDERÓN COMPLEX INTERPOLATION OF MORREY SPACES
}

\author{
DENNY HAKIM \\ Faculty of Mathematics and Natural Sciences, \\ Institut Teknologi Bandung, Bandung 40132, Indonesia. \\ dhakim@math.itb.ac.id
}

\begin{abstract}
In this note we will discuss some results related to complex interpolation of Morrey spaces. We first recall the Riesz-Thorin interpolation theorem in Section 1. After that, we discuss a partial generalization of this theorem in Morrey spaces proved in [19]. We also discuss non-interpolation property of Morrey spaces given in $[3,17]$. In Section 3, we recall the definition of Calderón's complex interpolation method and the description of complex interpolation of Lebesgue spaces. In Section 4 , we discuss the description of complex interpolation of Morrey spaces given in $[6,10,14,15]$. Finally, we discuss the description of complex interpolation of subspaces of Morrey spaces in the last section. This note is a summary of the current research about interpolation of Morrey spaces, generalized Morrey spaces, and their subspaces in $[6,9,10,11,12,14,15]$.
\end{abstract}

Key words and phrases: Morrey spaces, generalized Morrey spaces, complex interpolation.

\section{The Riesz-ThORIN InTERPolation THEOREM}

We first recall the definition of Lebesgue spaces. Let $1 \leq p \leq \infty$. The Lebesgue space $L^{p}=L^{p}\left(\mathbb{R}^{n}\right)$ is defined to be the set of all measurable function $f$ on $\mathbb{R}^{n}$ such that the norm

$$
\|f\|_{L^{p}}:= \begin{cases}\left(\int_{\mathbb{R}^{n}}|f(x)|^{p} d x\right)^{\frac{1}{p}}, & 1 \leq p<\infty, \\ \inf \left\{M>0:|f(x)| \leq M \text { a.e } x \in \mathbb{R}^{n}\right\}, & p=\infty\end{cases}
$$

is finite. An example of $L^{p}$-function is the simple function

$$
f=\sum_{j=1}^{k} a_{j} \chi_{A_{j}}
$$

2020 Mathematics Subject Classification: 10406, 2183, 11100 Received: 05-10-2018, accepted: 19-12-2018. 
where $a_{j} \in \mathbb{C}$ and $\left\{A_{j}\right\}_{j=1}^{k}$ is a collection of disjoint subsets of $\mathbb{R}^{n}$ with finite measure. In this case,

$$
\|f\|_{L^{p}}= \begin{cases}\left(\sum_{j=1}^{k}\left|a_{j}\right|^{p}\left|A_{j}\right|\right)^{\frac{1}{p}}, & p<\infty, \\ \max _{j=1,2, \ldots, k}\left|a_{j}\right|, & p=\infty .\end{cases}
$$

Note that the $L^{p}$ space is a Banach space with the norm defined in (1) Moreover, we also have the log-convexity property of $L^{p}$-norm as follows.

Lemma 1.1. [7, Exercise 1.1.16] Let $0 \leq \theta \leq 1,1 \leq p_{0}, p_{1} \leq \infty$, and $\frac{1}{p}=\frac{1-\theta}{p_{0}}+\frac{\theta}{p_{1}}$. Then for every $f \in L^{p_{0}} \cap L^{p_{1}}$ we have

$$
\|f\|_{L^{p}} \leq\|f\|_{L^{p_{0}}}^{1-\theta}\|f\|_{L^{p_{1}}}^{\theta} .
$$

Proof. Without loss of generality, assume that $p_{0}, p_{1}<\infty$. Since

$$
\frac{1}{p_{0} /((1-\theta) p)}+\frac{1}{p_{1} /(\theta p)}=1
$$

we have

$$
\|f\|_{L^{p}}^{p}=\int_{\mathbb{R}^{n}}|f(x)|^{p(1-\theta)}|f(x)|^{p \theta} d x \leq\|f\|_{L^{p_{0}}}^{p(1-\theta)}\|f\|_{L^{p_{1}}}^{p \theta} .
$$

Taking $p$ th root gives (2).

Note that, Lemma 1.1 can be viewed as the inclusion $L^{p_{0}} \cap L^{p_{1}} \subseteq L^{p}$. A complement to this result is the following lemma.

Lemma 1.2. Keep the same assumption as in Lemma 1.1. Then

$$
L^{p} \subseteq L^{p_{0}}+L^{p_{1}} .
$$

Here, $L^{p_{0}}+L^{p_{1}}$ is defined to be the set of all functions $f$ for which $f=f_{0}+f_{1}$ for some $f_{0} \in L^{p_{0}}$ and $f_{1} \in L^{p_{1}}$.

Proof. Without loss of generality, assume that $1 \leq p_{0}<p_{1}<\infty$. Let $f \in L^{p}$. Define $f_{0}:=f \chi_{\{|f| \geq 1\}}$ and $f_{1}:=f-f_{0}$. Since $p_{0}<p_{1}$, we have $p_{0}<p<p_{1}$, so

$$
\int_{\mathbb{R}^{n}}\left|f_{0}(x)\right|^{p_{0}} d x \leq \int_{\mathbb{R}^{n}}\left|f_{0}(x)\right|^{p_{0}-p}|f(x)|^{p} d x \leq\|f\|_{L^{p}}^{p}<\infty
$$

and

$$
\int_{\mathbb{R}^{n}}\left|f_{1}(x)\right|^{p_{1}} d x \leq \int_{\mathbb{R}^{n}}\left|f_{1}(x)\right|^{p_{1}-p}|f(x)|^{p} d x \leq\|f\|_{L^{p}}^{p}<\infty .
$$

Therefore, $f_{0} \in L^{p_{0}}$ and $f_{1} \in L^{p_{1}}$. Since $f=f_{0}+f_{1}$, we conclude that $f \in$ $L^{p_{0}}+L^{p_{1}}$.

As a preparation for proving the Riesz-Thorin interpolation theorem, we prove Hadamard's three lines lemma. 
Lemma 1.3. Let $S:=\{z \in \mathbb{C}: 0<\operatorname{Re}(z)<1\}$ and $\bar{S}$ be its closure. Let $F$ be any continuous function on $\bar{S}$ such that $F$ is holomorphic in $S$ and $F$ is bounded on $\bar{S}$. Then, for every $\theta \in(0,1)$ and $s \in \mathbb{R}$, we have

$$
|F(\theta+i s)| \leq\left(\sup _{t \in \mathbb{R}}|F(i t)|\right)^{1-\theta}\left(\sup _{t \in \mathbb{R}}|F(1+i t)|\right)^{\theta} .
$$

Proof. Let $M_{0}:=\sup _{t \in \mathbb{R}}|F(i t)|, M_{1}:=\sup _{t \in \mathbb{R}}|F(1+i t)|$, and $M:=\sup _{z \in \bar{S}}|F(z)|$. Define

Since

$$
G(z):=\frac{F(z)}{M_{0}^{1-z} M_{1}^{z}} \quad \text { and } \quad G_{n}(z):=G(z) e^{\frac{z^{2}-1}{n}}(n \in \mathbb{N}) .
$$

we have

$$
\left|M_{0}^{1-z} M_{1}^{z}\right|=M_{0}^{1-\operatorname{Re}(z)} M_{1}^{\operatorname{Re}(z)} \geq \min \left(1, M_{0}\right) \cdot \min \left(1, M_{1}\right),
$$

$$
|G(z)| \leq \frac{|F(z)|}{\left|M_{0}^{1-z} M_{1}^{z}\right|} \leq \frac{M}{\min \left(1, M_{0}\right) \cdot \min \left(1, M_{1}\right)}=: C .
$$

Consequently, for every $\sigma \in(0,1)$ and $r \in \mathbb{R}$, we have

$$
\left|G_{n}(\sigma+i r)\right|=|G(\sigma+i r)| e^{\frac{\sigma^{2}-r^{2}-1}{n}} \leq C e^{\frac{\sigma^{2}-1}{n}} e^{-\frac{r^{2}}{n}} \leq C e^{-\frac{r^{2}}{n}} .
$$

Therefore, $\lim _{|r| \rightarrow \infty} G_{n}(\sigma+i r)=0$ unifrormly over $\sigma \in[0,1]$. Hence, we can choose $R=R(n)>0$ such that for every $r \geq R$ and $\sigma \in[0,1]$, we have

$$
\left|G_{n}(\sigma+i r)\right| \leq 1
$$

Observe that for every $r \in \mathbb{R}$, we have

and

$$
\left|G_{n}(i r)\right|=\frac{|F(i r)|}{\left|M_{0}^{1-i r} M_{1}^{i r}\right|} e^{\frac{-r^{2}-1}{n}} \leq \frac{M_{0}}{M_{0}}=1
$$

$$
\left|G_{n}(1+i r)\right|=\frac{|F(1+i r)|}{\left|M_{0}^{1-i r} M_{1}^{i r}\right|} e^{\frac{-r^{2}}{n}} \leq \frac{M_{1}}{M_{1}}=1 .
$$

By the maximum modulus principle and (3)-(5), we have

$$
\left|G_{n}(z)\right| \leq 1
$$

whenever $0 \leq \operatorname{Re}(z) \leq 1$ and $-R \leq \operatorname{Im}(z) \leq R$. Combining (3) and (6), we have

$$
\left|G_{n}(z)\right| \leq 1
$$

for every $z \in \bar{S}$. Taking $n \rightarrow \infty$, we get

$$
|G(z)| \leq 1
$$

for every $z \in \bar{S}$. This implies

$$
|F(\theta+i s)|=|G(\theta+i s)| M_{0}^{1-\theta} M_{1}^{\theta} \leq M_{0}^{1-\theta} M_{1}^{\theta},
$$

as desired.

We now state and prove the Riesz-Thorin interpolation theorem as follows. 
Theorem 1.4. Let $0 \leq \theta \leq 1$ and $1 \leq p_{0}, p_{1}, q_{0}, q_{1} \leq \infty$. Suppose that $T$ is a bounded linear operator from $L^{p_{0}}$ to $L^{q_{0}}$ and $L^{p_{1}}$ to $L^{q_{1}}$. Then $T$ is bounded from $L^{p}$ to $L^{q}$, where $p$ and $q$ are defined by

$$
\frac{1}{p}:=\frac{1-\theta}{p_{0}}+\frac{\theta}{p_{1}} \text { and } \quad \frac{1}{q}:=\frac{1-\theta}{q_{0}}+\frac{\theta}{q_{1}} .
$$

Moreover,

$$
\|T\|_{L^{p} \rightarrow L^{q}} \leq\|T\|_{L^{p_{0}} \rightarrow L^{q_{0}}}^{1-\theta}\|T\|_{L^{p_{1}} \rightarrow L^{q_{1}}}^{\theta} .
$$

Proof. The proof follows the idea in [7]. We only handle the case where $p_{0}$ and $p_{1}$ are finite. Let $M_{0}:=\|T\|_{L^{p_{0}} \rightarrow L^{q_{0}}}$ and $M_{1}:=\|T\|_{L^{p_{1}} \rightarrow L^{q_{1}}}$. Let $f$ be a simple function and write

$$
f=\sum_{j=1}^{k} a_{j} e^{i \alpha_{j}} \chi_{A_{j}}
$$

where $a_{j}>0, \alpha_{j} \in \mathbb{R}$, and $\left\{A_{j}\right\}_{j=1}^{k}$ is a collection of pairwise disjoint subsets of $\mathbb{R}^{n}$ with $\left|A_{j}\right|<\infty$. Note that

$$
\|T f\|_{L^{q}}=\sup _{g \text { is simple, }\|g\|_{L^{q^{\prime}}}=1}\left|\int_{\mathbb{R}^{n}} T f(x) g(x) d x\right| .
$$

Now, let $g$ be fixed and write

$$
g=\sum_{\ell=1}^{m} b_{\ell} e^{i \beta_{\ell}} \chi_{B_{\ell}}
$$

where $b_{\ell}>0, \beta_{\ell} \in \mathbb{R}$, and $\left\{B_{\ell}\right\}_{\ell=1}^{m}$ is a collection of pairwise disjoint subsets of $\mathbb{R}^{n}$ with finite measure. Then, by linearity of $T$, we have

$$
\int_{\mathbb{R}^{n}} T f(x) g(x) d x=\sum_{j=1}^{k} \sum_{\ell=1}^{m} a_{j} e^{i \alpha_{j}} b_{\ell} e^{i \beta_{\ell}} \int_{\mathbb{R}^{n}} T \chi_{A_{j}}(x) \chi_{B_{\ell}}(x) d x .
$$

Let $S:=\{z \in \mathbb{C}: 0<\operatorname{Re}(z)<1\}$ and $\bar{S}$ be its closure. For every $z \in \bar{S}$, define

$$
F(z):=\sum_{j=1}^{k} \sum_{\ell=1}^{m} a_{j}^{\frac{p(1-z)}{p_{0}}+\frac{p z}{p_{1}}} e^{i \alpha_{j}} b_{\ell}^{\frac{q^{\prime}(1-z)}{q_{0}^{\prime}}+\frac{q^{\prime} z}{q_{1}^{\prime}}} e^{i \beta_{\ell}} \int_{\mathbb{R}^{n}} T \chi_{A_{j}}(x) \chi_{B_{\ell}}(x) d x,
$$

where $q_{0}^{\prime}:=\frac{q_{0}}{q_{0}-1}, q_{1}^{\prime}:=\frac{q_{1}}{q_{1}-1}$, and $q^{\prime}:=\frac{q}{q-1}$. Since $a_{j}$ and $b_{\ell}$ are positive, we see that $F$ is continuous on $\bar{S}$ and $F$ is holomorphic in $S$. Morever, by (7) and (9), we have

$$
F(\theta)=\int_{\mathbb{R}^{n}} T f(x) g(x) d x .
$$


By Hölder's inequality and the boundedness of $T$ from $L^{p_{0}}$ to $L^{q_{0}}$, for every $z \in \bar{S}$, we have

$$
\begin{aligned}
& |F(z)| \leq \sum_{j=1}^{k} \sum_{\ell=1}^{m} a_{j}^{\frac{p(1-\operatorname{Re}(z))}{p_{0}}+\frac{p \operatorname{Re}(z)}{p_{1}}} b_{\ell}^{\frac{q^{\prime}(1-\operatorname{Re}(z))}{q_{0}^{\prime}}+\frac{q^{\prime} \operatorname{Re}(z)}{q_{1}^{\prime}}}\left|\int_{\mathbb{R}^{n}} T \chi_{A_{j}}(x) \chi_{B_{\ell}}(x) d x\right| \\
& \leq \sum_{j=1}^{k} \sum_{\ell=1}^{m}\left(a_{j}^{\frac{p}{p_{0}}}+a_{j}^{\frac{p}{p_{1}}}\right)\left(b_{\ell}^{\frac{q^{\prime}}{q_{0}^{\prime}}}+b_{\ell}^{\frac{q^{\prime}}{q_{1}^{\prime}}}\right) M_{0}\left|A_{j}\right|^{\frac{1}{p_{0}}}\left|B_{\ell}\right|^{\frac{1}{q_{0}^{\prime}}} .
\end{aligned}
$$

Therefore, $\sup _{z \in \bar{S}}|F(z)|<\infty$. Note that $F(z)$ can be rewritten as

$$
F(z)=\int_{\mathbb{R}^{n}} T f_{z}(x) g_{z}(x) d x
$$

where

$$
f_{z}:=\sum_{j=1}^{k} a_{j}^{\frac{p(1-z)}{p_{0}}+\frac{p z}{p_{1}}} e^{i \alpha_{j}} \chi_{A_{j}} \quad \text { and } \quad g_{z}:=\sum_{\ell=1}^{m} b_{\ell}^{\frac{q^{\prime}(1-z)}{q_{0}^{\prime}}+\frac{q^{\prime} z}{q_{1}^{\prime}}} e^{i \beta_{\ell}} \chi_{B_{\ell}} .
$$

By Hölder's inequality and the boundedness of $T$ from $L^{p_{0}}$ to $L^{q_{0}}$, for every $t \in \mathbb{R}$, we have

$$
|F(i t)| \leq\left\|T f_{i t}\right\|_{L^{q_{0}}}\left\|g_{i t}\right\|_{L^{q_{0}^{\prime}}} \leq M_{0}\left\|f_{i t}\right\|_{L^{p_{0}}}\left\|g_{i t}\right\|_{L^{q_{0}^{\prime}}} .
$$

Since $A_{j}$ 's are pairwise disjoint, we have

$$
\left\|f_{i t}\right\|_{L^{p_{0}}}^{p_{0}}=\sum_{j=1}^{k} a_{j}^{p}\left|A_{j}\right|=\|f\|_{L^{p}}^{p}
$$

so $\left\|f_{i t}\right\|_{L^{p_{0}}}=\|f\|_{L^{p}}^{p / p_{0}}$. Likewise, $\left\|g_{i t}\right\|_{L^{q_{0}^{\prime}}}=\|g\|_{L^{q^{\prime}}}^{q^{\prime}}=1$. Combining these calculations with (11), we obtain

$$
|F(i t)| \leq M_{0}\|f\|_{L^{p}}^{p / p_{0}} .
$$

Similarly,

$$
|F(1+i t)| \leq M_{1}\|f\|_{L^{p}}^{p / p_{1}} .
$$

By the three-lines lemma and (12)-(13), we have

$$
|F(\theta)| \leq M_{0}^{1-\theta} M_{1}^{\theta}\|f\|_{L^{p}}^{\frac{p(1-\theta)}{p_{0}}}\|f\|_{L^{p}}^{\frac{p \theta}{p_{1}}}=M_{0}^{1-\theta} M_{1}^{\theta}\|f\|_{L^{p}} .
$$

Combining this inequality with (8) and (10), we have

$$
\|T f\|_{L^{q}} \leq M_{0}^{1-\theta} M_{1}^{\theta}\|f\|_{L^{p}}
$$

for every simple function $f$. Finally, (14) can be extended for all $f \in L^{p}$ by using the density of simple functions in $L^{p}$. 


\section{INTERPOLATION OF LINEAR OPERATORS IN MORREY SPACES}

Definition 2.1. For $1 \leq q \leq p<\infty$, the Morrey space $\mathcal{M}_{q}^{p}=\mathcal{M}_{q}^{p}\left(\mathbb{R}^{n}\right)$ is defined to be the set of all functions $f \in L_{\mathrm{loc}}^{q}\left(\mathbb{R}^{n}\right)$ such that

$$
\|f\|_{\mathcal{M}_{q}^{p}}:=\sup _{a \in \mathbb{R}^{n}, R>0}|B(a, R)|^{\frac{1}{p}}\left(\frac{1}{|B(a, R)|} \int_{B(a, R)}|f(x)|^{q} d x\right)^{\frac{1}{q}}
$$

is finite.

\section{Remark:}

(1) For $p=q$, we have $\mathcal{M}_{q}^{p}=L^{q}$.

(2) If $1 \leq q<p<\infty$, then $f(x):=|x|^{-\frac{n}{p}} \in \mathcal{M}_{q}^{p} \backslash L^{p}$.

G. Stampacchia proved the following extension of the Riesz-Thorin interpolation theorem.

Theorem 2.2. Let $\theta \in(0,1), 1 \leq p_{0}, p_{1}<\infty, 1 \leq s_{0} \leq r_{0}<\infty$, and $1 \leq s_{1} \leq$ $r_{1}<\infty$. Define $p, r$, and $s$ by

$$
\left(\frac{1}{p}, \frac{1}{r}, \frac{1}{s}\right):=(1-\theta)\left(\frac{1}{p_{0}}, \frac{1}{r_{0}}, \frac{1}{s_{0}}\right)+\theta\left(\frac{1}{p_{1}}, \frac{1}{r_{1}}, \frac{1}{s_{1}}\right) .
$$

If $T$ is a bounded linear operator from $L^{p_{0}}$ to $\mathcal{M}_{s_{0}}^{r_{0}}$ and from $L^{p_{1}}$ to $\mathcal{M}_{s_{1}}^{r_{1}}$, then $T$ is bounded from $L^{p}$ to $\mathcal{M}_{s}^{r}$.

Unfortunately, if the domain of the operator $T$ is Morrey spaces, there are some counterexamples given by A. Ruiz and L. Vega [17] for the case $n>1$ and by O. Blasco et al. in [3] for the case $n=1$. Let us recall the result in [3].

Theorem 2.3. [3] Let $n=1, \theta \in(0,1)$, and $1<q_{1}<q_{0}$. Define

$$
\frac{1}{q}:=\frac{1-\theta}{q_{0}}+\frac{\theta}{q_{1}}, r_{0}:=\frac{2}{\min \left(\frac{1}{q_{0}}+\frac{2}{q_{1}} \cdot 2\right)}, r_{1}:=q_{1} \text {, and } \frac{1}{r}:=\frac{1-\theta}{r_{0}}+\frac{\theta}{r_{1}} .
$$

Then there exists a bounded linear operator $T$ from $L^{q_{0}}=\mathcal{M}_{q_{0}}^{q_{0}}$ to $L^{r_{0}}$ and from $\mathcal{M}_{q_{1}}^{q_{0}}$ to $L^{r_{1}}$ such that $T$ is not bounded from $\mathcal{M}_{q}^{q_{0}}$ to $L^{r}$.

Proof. According to the definition of $q$, we know that $q_{1}<q<q_{0}$. Hence, we may choose

$$
\beta>\frac{\frac{1}{q_{0}}}{\frac{1}{q}-\frac{1}{q_{0}}}
$$

Let $N_{0} \in \mathbb{N}$ be such that

$$
\frac{\beta+1}{\log 2}<\frac{N+1}{\log N}
$$

for every $N \in \mathbb{N} \cap\left[N_{0}, \infty\right)$. Let $N \in \mathbb{N} \cap\left[N_{0}, \infty\right)$ be fixed. We define

$$
I_{j}^{N}:=\left[N !+j N^{\beta}, N !+j N^{\beta}+1\right]
$$


where $j=0,1, \ldots, N-1$ and set $E_{N}:=\cup_{j=0}^{N-1} I_{j}^{N}$. Observe that the choice of $\beta$ allows $\left\{E_{N}\right\}_{N=1}^{\infty}$ to be disjoint. Note that $r_{0}<r_{1}$, so $r_{0}<r<r_{1}$. Therefore, we may choose

$$
\gamma \in\left(\frac{2}{r_{1}}, \frac{2}{r}\right) .
$$

With this choice of $\gamma$, we construct an operator $T$ by the formula

$$
T f(x):=\sum_{N=N_{0}}^{\infty} N^{-\gamma} \chi_{E_{N}}(x) f(x)
$$

for every measurable function $f$. By the Hölder inequality, for every $f \in L^{q_{0}}$ we have

$$
\begin{aligned}
\|T f\|_{L^{r_{0}}} & \leq\left(\sum_{N=N_{0}}^{\infty} N^{-\gamma r_{0}}\left|E_{N}\right|^{1-\frac{r_{0}}{q_{0}}}\left(\int_{E_{N}}|f(x)|^{q_{0}}\right)^{\frac{r_{0}}{q_{0}}}\right)^{\frac{1}{r_{0}}} \\
& \leq\left(\sum_{N=N_{0}}^{\infty} N^{-\gamma r_{0}+1-\frac{r_{0}}{q_{0}}}\right)^{\frac{1}{r_{0}}}\|f\|_{L^{q_{0}}} .
\end{aligned}
$$

It follows from (17) that

$$
-\gamma r_{0}+1-\frac{r_{0}}{q_{0}}<-\frac{2 r_{0}}{r_{1}}+1-\frac{r_{0}}{q_{0}}=1-r_{0}\left(\frac{2}{q_{1}}+\frac{1}{q_{0}}\right) \leq-1 .
$$

Consequently,

$$
\|T f\|_{L^{r_{0}}} \leq C_{0}\|f\|_{L^{q_{0}}}
$$

for some constant $C_{0}>0$. We now show that

$$
\|T f\|_{L^{r_{1}}} \leq C_{1}\|f\|_{\mathcal{M}_{q_{1}}^{q_{0}}}
$$

for some $C_{1}>0$ and for every $f \in \mathcal{M}_{q_{1}}^{q_{0}}$. Since $\left\{E_{N}\right\}_{N=N_{0}}^{\infty}$ is a collection of disjoint sets and $q_{1}=r_{1}$, we get

$$
\|T f\|_{L^{r_{1}}} \leq\left(\sum_{N=N_{0}}^{\infty} N^{-\gamma r_{1}} \sum_{j=0}^{N-1} \int_{I_{j}^{N}}|f(x)|^{r_{1}} d x\right)^{\frac{1}{r_{1}}} .
$$

Combining (19) and

$$
\int_{I_{j}^{N}}|f(x)|^{q_{1}} d x \leq\left|I_{j}^{N}\right|^{1-\frac{q_{1}}{q_{0}}}\|f\|_{\mathcal{M}_{q_{1}}^{q_{0}}}^{q_{1}}=\|f\|_{\mathcal{M}_{q_{1}}^{q_{0}}}^{q_{1}}
$$

for each $j=0,1, \ldots, N-1$, we get

$$
\|T f\|_{L^{r_{1}}} \leq\left(\sum_{N=N_{0}}^{\infty} N^{1-\gamma r_{1}}\right)^{\frac{1}{r_{1}}}\|f\|_{\mathcal{M}_{q_{1}}^{q_{0}}} .
$$

According to (17), we have

$$
1-\gamma r_{1}<1-\frac{2}{r_{1}} r_{1}=-1
$$


SO

$$
\sum_{N=N_{0}}^{\infty} N^{1-\gamma r_{1}}<\infty
$$

This implies (18). The proof of the unboundedness of $T$ from $\mathcal{M}_{q}^{q_{0}}$ to $L^{r}$ goes as follows. Define

$$
f_{0}:=\sum_{N=N_{0}}^{\infty} \chi_{E_{N}}
$$

Note that, for every $N \in \mathbb{N}$, we have

$$
\begin{aligned}
\left\|\chi_{E_{N}}\right\|_{\mathcal{M}_{1}^{\frac{q_{0}}{q}}} & =\sup _{I \subseteq \mathbb{R}}|I|^{\frac{q}{q_{0}}} \frac{\left|I \cap E_{N}\right|}{|I|} \\
& \lesssim\left((N-1) N^{\beta}+1\right)^{\frac{q}{q_{0}}} \frac{N}{(N-1) N^{\beta}+1} \\
& \lesssim\left(N^{\beta+1}\right)^{\frac{q}{q_{0}}} \frac{1}{N^{\beta}}=N^{\frac{q(\beta+1)}{q_{0}}-\beta} .
\end{aligned}
$$

Let $J_{N}:=\left(N_{0} !, N !+(N-1) N^{\beta}+1\right)$ for every $N \in \mathbb{N} \cap\left[N_{0}, \infty\right)$. Since

$$
\left\|f_{0}\right\|_{\mathcal{M}_{q}^{q_{0}}}^{q}=\left\|f_{0}^{q}\right\|_{\mathcal{M}_{1}^{\frac{q_{0}}{q}}}=\left\|f_{0}\right\|_{\mathcal{M}_{1}^{\frac{q_{0}}{q}}}
$$

we have

$$
\begin{aligned}
\left\|f_{0}\right\|_{\mathcal{M}_{q}^{q_{0}}}^{q} & =\sup _{I \in \mathcal{I}(\mathbb{R})}|I|^{\frac{q}{q_{0}}-1} \int_{I} \sum_{N=N_{0}}^{\infty} \chi_{E_{N}}(y) d y \\
& \lesssim \max _{M \in \mathbb{N}}\left\{\left|J_{M}\right|^{\frac{q}{q_{0}}-1} \int_{J_{M}} \sum_{N=N_{0}}^{M} \chi_{E_{N}}(y) d y,\left\|\chi_{E_{M}}\right\|_{\mathcal{M}_{1}^{\frac{q_{0}}{q}}}\right\} \\
& \lesssim \max _{M \in \mathbb{N}}\left\{\frac{M^{2}}{\left(M !+(M-1) M^{\beta}+1-N_{0} !\right)^{1-\frac{q}{q_{0}}}}, M^{\frac{q(\beta+1)}{q_{0}}-\beta}\right\} .
\end{aligned}
$$

It follows from (15) that $\frac{q(\beta+1)}{q_{0}}-\beta<0$. This implies

$$
\left\|f_{0}\right\|_{\mathcal{M}_{q}^{q_{0}}}<\infty \text {. }
$$

On the other hand, we claim

$$
\left\|T f_{0}\right\|_{L^{r}}=\infty
$$

Indeed, (20) follows from

$$
\left\|T f_{0}\right\|_{L^{r}}=\left(\sum_{N=N_{0}}^{\infty} N^{-\gamma r}\left|E_{N}\right|\right)^{\frac{1}{r}}=\left(\sum_{N=N_{0}}^{\infty} N^{1-\gamma r}\right)^{\frac{1}{r}}
$$

and $1-\gamma r>-1$. This ends the proof of Theorem 2.3.

In view of Theorem 2.3, the Riesz-Thorin theorem can not be naturally generalized to Morrey spaces. However, by adding some mild assumptions, there are 
recent researches about complex interpolation interpolation of Morrey spaces and their generalization (see $[6,9,10,11,12,13,14,15]$ ).

\section{CALderón's COMplex interpolation method}

In this section we recall the complex interpolation method introduced by Calderón in [4]. We follow the terminology and presentation in $[1,4,12]$. In Subsections 3.1 and 3.2, we recall the definition of Calderón's first and second complex interpolation method. For the proof of our results in the next section, we shall discuss the Calderón product of Banach spaces in Section 3.3.

3.1. The first complex interpolation method. A pair $\left(X_{0}, X_{1}\right)$ is said to be a compatible couple of Banach spaces if there exists a Hausdorff topological vector space $Z$ such that $X_{0}$ and $X_{1}$ are subspaces of $Z$ and that the embedding of $X_{0}$ and $X_{1}$ into $Z$ is continous. From now on, let $\bar{S}:=\{z \in \mathbb{C}: 0 \leq \operatorname{Re}(z) \leq 1\}$ and $S$ be its interior.

Definition 3.1 (Calderón's first complex interpolation functor). Let $\left(X_{0}, X_{1}\right)$ be a compatible couple of Banach spaces. Define $\mathcal{F}\left(X_{0}, X_{1}\right)$ as the set of all continuous functions $F: \bar{S} \rightarrow X_{0}+X_{1}$ such that

(1) $\sup _{\bar{S}}\|F(z)\|_{X_{0}+X_{1}}<\infty$,

$$
z \in \bar{S}
$$

(2) $F$ is holomorphic on $S$,

(3) the functions $t \in \mathbb{R} \mapsto F(j+i t) \in X_{j}$ are bounded and continuous on $\mathbb{R}$ for $j=0,1$.

The norm on $\mathcal{F}\left(X_{0}, X_{1}\right)$ is defined by

$$
\|F\|_{\mathcal{F}\left(X_{0}, X_{1}\right)}:=\max \left\{\sup _{t \in \mathbb{R}}\|F(i t)\|_{X_{0}}, \sup _{t \in \mathbb{R}}\|F(1+i t)\|_{X_{1}}\right\} .
$$

Definition 3.2 (Calderón's first complex interpolation spaces). Let $\theta \in(0,1)$ and $\left(X_{0}, X_{1}\right)$ be a compatible couple of Banach spaces. The complex interpolation space $\left[X_{0}, X_{1}\right]_{\theta}$ with respect to $\left(X_{0}, X_{1}\right)$ is defined by

$$
\left[X_{0}, X_{1}\right]_{\theta}:=\left\{f \in X_{0}+X_{1}: f=F(\theta) \text { for some } F \in \mathcal{F}\left(X_{0}, X_{1}\right)\right\}
$$

The norm on $\left[X_{0}, X_{1}\right]_{\theta}$ is defined by

$$
\|f\|_{\left[X_{0}, X_{1}\right]_{\theta}}:=\inf \left\{\|F\|_{\mathcal{F}\left(X_{0}, X_{1}\right)}: f=F(\theta) \text { for some } F \in \mathcal{F}\left(X_{0}, X_{1}\right)\right\} .
$$

The fact that $\left[X_{0}, X_{1}\right]_{\theta}$ is a Banach space can be seen in [4] and [1, Theorem 4.1.2]. When $X_{0}$ and $X_{1}$ are Lebesgue spaces, Calderón gave the following description of $\left[X_{0}, X_{1}\right]_{\theta}$.

Theorem 3.3. [4] Let $\theta \in(0,1), 1 \leq p_{0} \leq \infty$, and $1 \leq p_{1} \leq \infty$. Then

$$
\left[L^{p_{0}}, L^{p_{1}}\right]_{\theta}=L^{p}
$$

where $p$ is defined by

$$
\frac{1}{p}:=\frac{1-\theta}{p_{0}}+\frac{\theta}{p_{1}}
$$


Note that the Riesz-Thorin complex interpolation theorem can be seen as a corollary of Theorem 3.3 and the following Calderón's result.

Theorem 3.4. [4] Let $\theta \in(0,1)$. Let $\left(X_{0}, X_{1}\right)$ and $\left(Y_{0}, Y_{1}\right)$ be two compatible couples of Banach spaces. If $T$ is a bounded linear operator from $X_{j}$ to $Y_{j}$ for $j=0,1$, then $T$ is bounded from $\left[X_{0}, X_{1}\right]_{\theta}$ to $\left[Y_{0}, Y_{1}\right]_{\theta}$.

We also invoke the following useful lemma.

Lemma 3.5. [4], [1, Theorem 4.2.2] Let $\theta \in(0,1)$ and $\left(X_{0}, X_{1}\right)$ be a compatible couple of Banach spaces. Then we have $X_{0} \cap X_{1}$ is dense in $\left[X_{0}, X_{1}\right]_{\theta}$.

3.2. The second complex interpolation method. First let us recall the definition of Banach space-valued Lipschitz continuous functions. Let $X$ be a Banach space. Denote by $\operatorname{Lip}(\mathbb{R}, X)$ the set of all functions $f: \mathbb{R} \rightarrow X$ such that

$$
\|f\|_{\operatorname{Lip}(\mathbb{R}, X)}:=\sup _{-\infty<s<t<\infty} \frac{\|f(t)-f(s)\|_{X}}{|t-s|}
$$

is finite.

Definition 3.6. $[1,4]$ (Calderón's second complex interpolation functor) Let $\left(X_{0}, X_{1}\right)$ be a compatible couple of Banach spaces. Denote by $\mathcal{G}\left(X_{0}, X_{1}\right)$ the set of all continuous functions $G: \bar{S} \rightarrow X_{0}+X_{1}$ such that:

(1) $\sup _{z \in \bar{S}}\left\|\frac{G(z)}{1+|z|}\right\|_{X_{0}+X_{1}}<\infty$

(2) $G$ is holomorphic on $S$,

(3) the functions

$$
t \in \mathbb{R} \mapsto G(j+i t)-G(j) \in X_{j}
$$

are Lipschitz continuous on $\mathbb{R}$ for $j=0,1$.

The space $\mathcal{G}\left(X_{0}, X_{1}\right)$ is equipped with the norm

$$
\|G\|_{\mathcal{G}\left(X_{0}, X_{1}\right)}:=\max \left\{\|G(i \cdot)\|_{\operatorname{Lip}\left(\mathbb{R}, X_{0}\right)},\|G(1+i \cdot)\|_{\operatorname{Lip}\left(\mathbb{R}, X_{1}\right)}\right\} .
$$

Definition 3.7. $[1,4]$ (Calderón's second complex interpolation space) Let $\theta \in$ $(0,1)$. The second complex interpolation space $\left[X_{0}, X_{1}\right]^{\theta}$ with respect to $\left(X_{0}, X_{1}\right)$ is defined to be the set of all $f \in X_{0}+X_{1}$ such that $f=G^{\prime}(\theta)$ for some $G \in \mathcal{G}\left(X_{0}, X_{1}\right)$. The norm on $\left[X_{0}, X_{1}\right]^{\theta}$ is defined by

$$
\|f\|_{\left[X_{0}, X_{1}\right]^{\theta}}:=\inf \left\{\|G\|_{\mathcal{G}\left(X_{0}, X_{1}\right)}: f=G^{\prime}(\theta) \text { for some } G \in \mathcal{G}\left(X_{0}, X_{1}\right)\right\} .
$$

The relation between the second complex interpolation and the interpolation of linear operators is given as follows.

Theorem 3.8 (Calderón, 1964). Let $\theta \in(0,1)$ and $j \in\{0,1\}$. Suppose that $T$ is a bounded linear operator from $X_{j}$ to $Y_{j}$. Then, $T$ is bounded from $\left[X_{0}, X_{1}\right]^{\theta}$ to $\left[Y_{0}, Y_{1}\right]^{\theta}$.

We now describe the second complex interpolation of Lebesgue spaces. 
Theorem 3.9 (Calderón, 1964). Let $\theta \in(0,1), 1 \leq p_{0}, p_{1} \leq \infty$, and $\frac{1}{p}:=\frac{1-\theta}{p_{0}}+\frac{\theta}{p_{1}}$. Then

$$
\left[L^{p_{0}}, L^{p_{1}}\right]^{\theta}=L^{p}
$$

The relation between the inclusion and the second complex interpolation spaces is given as follows.

Lemma 3.10. [11, Lemma 2.8] If $X_{0} \hookrightarrow Y_{0}$ and $X_{1} \hookrightarrow Y_{1}$, then

$$
\left[X_{0}, X_{1}\right]^{\theta} \hookrightarrow\left[Y_{0}, Y_{1}\right]^{\theta} \text {. }
$$

Proof. Let $f \in\left[X_{0}, X_{1}\right]^{\theta}$. Then $f=G^{\prime}(\theta)$ for some $G \in \mathcal{G}\left(X_{0}, X_{1}\right)$. By using the following inequalities

$$
\left\|x_{0}\right\|_{Y_{0}} \lesssim\left\|x_{0}\right\|_{X_{0}}, \quad\left\|x_{1}\right\|_{Y_{1}} \lesssim\left\|x_{1}\right\|_{X_{1}}, \quad \text { and } \quad\|x\|_{Y_{0}+Y_{1}} \lesssim\|x\|_{X_{0}+X_{1}},
$$

for every $x_{0} \in X_{0}, x_{1} \in X_{1}$, and $x \in X_{0}+X_{1}$, we can show that $G \in \mathcal{G}\left(Y_{0}, Y_{1}\right)$. Thus, $f \in\left[Y_{0}, Y_{1}\right]^{\theta}$.

The relation between the first and second complex interpolation functors is given in the following lemma:

Lemma 3.11. [10, Lemma 2.4] For $G \in \mathcal{G}\left(X_{0}, X_{1}\right), z \in \bar{S}$, and $k \in \mathbb{N}$, define

$$
H_{k}(z):=\frac{G\left(z+2^{-k} i\right)-G(z)}{2^{-k} i} .
$$

Then we have $H_{k}(\theta) \in\left[X_{0}, X_{1}\right]_{\theta}$.

Proof. We give a simplified proof of [10, Lemma 2.4]. The proof is adapted from [11]. The continuity and holomorphicity of $H_{k}$ is a consequence of the corresponding property of $G$. Let $j \in\{0,1\}$ be fixed. Since $t \in \mathbb{R} \mapsto G(j+i t) \in X_{j}$ is Lipschitzcontinuous, we see that $t \in \mathbb{R} \mapsto H_{k}(j+i t) \in X_{j}$ is bounded and continuous on $\mathbb{R}$. Therefore, $H_{k} \in \mathcal{F}\left(X_{0}, X_{1}\right)$. Moreover,

$$
\begin{aligned}
\left\|H_{k}(\theta)\right\|_{\left[X_{0}, X_{1}\right]_{\theta}} & \leq\left\|H_{k}\right\|_{\mathcal{F}\left(X_{0}, X_{1}\right)} \\
& =\max _{j=0,1} \sup _{t \in \mathbb{R}}\left\|\frac{G\left(j+i\left(t+2^{-k}\right)\right)-G(j+i t)}{2^{-k} i}\right\|_{X_{j}} \\
& \leq\|G\|_{\mathcal{G}\left(X_{0}, X_{1}\right)}<\infty,
\end{aligned}
$$

as desired.

We shall also use the following useful connection between the first and second complex interpolation, obtained by Bergh [2].

Lemma 3.12. [2] Let $\left(X_{0}, X_{1}\right)$ be a compatible couple and $\theta \in(0,1)$. Then we have

$$
\left[X_{0}, X_{1}\right]_{\theta}=\overline{X_{0} \cap X_{1}}\left[X_{0}, X_{1}\right]^{\theta} .
$$


3.3. Calderón product. In order to describe the first complex interpolation spaces, sometimes it is easier to calculate the Calderón product of Banach lattices and applying the result of Sestakov in [18]. The definition of the Calderón product and Sestakov's lemma are given as follows.

Definition 3.13. Let $\theta \in(0,1)$ and $\left(X_{0}, X_{1}\right)$ be a compatible couple of Banach spaces of measurable functions in $\mathbb{R}^{n}$. The Calderón product $X_{0}{ }^{1-\theta} X_{1}{ }^{\theta}$ of $X_{0}$ and $X_{1}$ is defined by

$$
X_{0}{ }^{1-\theta} X_{1}{ }^{\theta}:=\bigcup_{f_{0} \in X_{0}, f_{1} \in X_{1}}\left\{f: \mathbb{R}^{n} \rightarrow \mathbb{C}:|f(x)| \leq\left|f_{0}(x)\right|^{1-\theta}\left|f_{1}(x)\right|^{\theta} \text { a.e. } x \in \mathbb{R}^{n}\right\} .
$$

For $f \in X_{0}{ }^{1-\theta} X_{1}{ }^{\theta}$, we define

$$
\begin{aligned}
& \|f\|_{X_{0}{ }^{1-\theta} X_{1}{ }^{\theta}} \\
& :=\inf \left\{\left\|f_{0}\right\|_{X_{0}}^{1-\theta}\left\|f_{1}\right\|_{X_{1}}^{\theta}: f_{0} \in X_{0}, f_{1} \in X_{1},|f(x)| \leq\left|f_{0}(x)\right|^{1-\theta}\left|f_{1}(x)\right|^{\theta} \text { a.e. } x \in \mathbb{R}^{n}\right\} .
\end{aligned}
$$

Theorem 3.14. Let $\theta \in(0,1)$ and $\left(X_{0}, X_{1}\right)$ be a compatible couple of Banach spaces of measurable functions in $\mathbb{R}^{n}$. Then $X_{0}^{1-\theta} X_{1}^{\theta}$ is a Banach space.

Proof. This result was due to Calderón [4]. For the convenience of the reader, we give the detailed proof. We first prove the triangle inequality in $X_{0}^{1-\theta} X_{1}^{\theta}$. Let $f, g \in X_{0}{ }^{1-\theta} X_{1}{ }^{\theta}$. Choose $\lambda \in\left(\|f\|_{X_{0}{ }^{1-\theta} X_{1} \theta}, \infty\right)$ and $\rho \in\left(\|g\|_{X_{0}{ }^{1-\theta} X_{1} \theta}, \infty\right)$. Then there is a decomposition

$$
|f(x)| \leq \lambda\left|f_{0}(x)\right|^{1-\theta}\left|f_{1}(x)\right|^{\theta}, \quad|g(x)| \leq\left.\rho g_{0}(x)\right|^{1-\theta}\left|g_{1}(x)\right|^{\theta}
$$

such that $f_{0}, g_{0} \in X_{0}$ and $f_{1}, g_{1} \in X_{1}$ have norm 1 . Observe that (24) is equivalent to:

$$
|f(x)| \leq(1-\theta) \varphi(x)^{-\theta} \lambda\left|f_{0}(x)\right|+\theta \varphi(x)^{1-\theta} \lambda\left|f_{1}(x)\right|
$$

and

$$
|g(x)| \leq(1-\theta) \varphi(x)^{-\theta} \rho\left|g_{0}(x)\right|+\theta \varphi(x)^{1-\theta} \rho\left|g_{1}(x)\right|
$$

for any measurable function $\varphi: \mathbb{R}^{n} \rightarrow(0, \infty)$. Since

$$
|f(x)+g(x)| \leq(1-\theta) \varphi^{-\theta}\left(\lambda\left|f_{0}(x)\right|+\rho\left|g_{0}(x)\right|\right)+\theta \varphi^{1-\theta}\left(\lambda\left|f_{1}(x)\right|+\rho\left|g_{1}(x)\right|\right),
$$

we conclude that

$$
|f(x)+g(x)| \leq\left(\lambda\left|f_{0}(x)\right|+\rho\left|g_{0}(x)\right|\right)^{1-\theta}\left(\lambda\left|f_{1}(x)\right|+\rho\left|g_{1}(x)\right|\right)^{\theta} .
$$

This implies that $\|f+g\|_{X_{0}^{1-\theta} X_{1} \theta} \leq\|f\|_{X_{0}^{1-\theta} X_{1} \theta}+\|g\|_{X_{0}^{1-\theta} X_{1}{ }^{\theta}}$.

The proof of completeness of $X_{0}{ }^{1-\theta} X_{1}{ }^{\theta}$ goes as follows. Let $\left\{f^{j}\right\}_{j=1}^{\infty} \subset$ $X_{0}{ }^{1-\theta} X_{1}{ }^{\theta}$ be a sequence satisfying

$$
\sum_{j=1}^{\infty}\left\|f^{j}\right\|_{X_{0}{ }^{1-\theta} X_{1}{ }^{\theta}}<\infty
$$

Let $\lambda_{j} \in\left(\|f\|_{X_{0}{ }^{1-\theta} X_{1}{ }^{\theta}}, \infty\right)$. Then, there exists $f_{0}^{j} \in X_{0}$ and $f_{1}^{j} \in X_{1}$ have norm 1 such that

$$
\left|f^{j}(x)\right| \leq \lambda_{j}\left|f_{0}^{j}(x)\right|^{1-\theta}\left|f_{1}^{j}(x)\right|^{\theta} .
$$


Then as before,

$$
\sum_{j=1}^{\infty}\left|f_{j}(x)\right| \leq\left(\sum_{j=1}^{\infty} \lambda_{j}\left|f_{0}^{j}(x)\right|\right)^{1-\theta}\left(\sum_{j=1}^{\infty} \lambda_{j}\left|f_{1}^{j}(x)\right|\right)^{\theta}
$$

Since $X_{0}$ and $X_{1}$ are Banach spaces, we see that $\sum_{j=1}^{\infty} \lambda_{j}\left|f_{0}^{j}\right|$ and $\sum_{j=1}^{\infty} \lambda_{j}\left|f_{1}^{j}\right|$ converge in $X_{0}$ and $X_{1}$, respectively. Consequently, $\sum_{j=1}^{\infty} f_{j}(x)$ converges absolutely for almost all $x \in \mathbb{R}^{n}$ and belongs to $X_{0}{ }^{1-\theta} X_{1}{ }^{\theta}$ together with the estimate

$$
\left\|\sum_{j=1}^{\infty} f_{j}\right\|_{X_{0}^{1-\theta} X_{1} \theta} \leq \sum_{j=1}^{\infty}\left\|f_{j}\right\|_{X_{0}^{1-\theta} X_{1} \theta}
$$

or more generally

$$
\left\|\sum_{j=J}^{\infty} f_{j}\right\|_{X_{0}{ }^{1-\theta} X_{1} \theta} \leq \sum_{j=J}^{\infty}\left\|f_{j}\right\|_{X_{0}{ }^{1-\theta} X_{1} \theta},(J \in \mathbb{N})
$$

which also yields that $\sum_{j=1}^{\infty} f_{j}$ converges in $X_{0}{ }^{1-\theta} X_{1}{ }^{\theta}$.

By virtue of the Hölder inequality and factorization, for $1 \leq p_{0}, p_{1} \leq \infty$

$$
\left(L^{p_{0}}\right)^{1-\theta}\left(L^{p_{1}}\right)^{\theta}=L^{p}
$$

where $p$ is defined by $\frac{1}{p}:=\frac{1-\theta}{p_{0}}+\frac{\theta}{p_{1}}$. We now recall the following result by Sestakov.

Lemma 3.15. [18] Let $\left(X_{0}, X_{1}\right)$ be a compatible couple of Banach spaces of measurable functions in $\mathbb{R}^{n}$. Then for every $\theta \in(0,1)$, we have

$$
\left[X_{0}, X_{1}\right]_{\theta}=\bar{X}_{0} \cap X_{1}{ }^{1-\theta} X_{1}^{\theta}
$$

\section{The DESCRIPTION OF COMPLEX INTERPOLATION OF MORREy SPACES}

In this section, we will discuss the first and second complex interpolation of Morrey spaces. The interpolation by using the first method can be found in $[6,9,10,15]$. Meanwhile, the result on the second complex interpolation is given by Lemarié-Rieusset [14]. The presentation in this section and Section 5 follows $[12]$.

4.1. The first complex interpolation of Morrey spaces. The first result about the description of the first complex interpolation of Morrey spaces was due to Cobos et al. [6]. 
Theorem 4.1. [6] Let $\theta \in(0,1), 1 \leq q_{0} \leq p_{0}<\infty$, and $1 \leq q_{1} \leq p_{1}<\infty$. Define $p$ and $q$ by

$$
\frac{1}{p}:=\frac{1-\theta}{p_{0}}+\frac{\theta}{p_{1}} \text { and } \frac{1}{q}:=\frac{1-\theta}{q_{0}}+\frac{\theta}{q_{1}},
$$

respectively. Then

$$
\left[\mathcal{M}_{q_{0}}^{p_{0}}, \mathcal{M}_{q_{1}}^{p_{1}}\right]_{\theta} \subseteq \mathcal{M}_{q}^{p} .
$$

Assuming $\frac{p_{0}}{q_{0}}=\frac{p_{1}}{q_{1}}$, Lu et al. [15] improved the description of $\left[\mathcal{M}_{q_{0}}^{p_{0}}, \mathcal{M}_{q_{1}}^{p_{1}}\right]_{\theta}$ in Theorem 4.1. Morever, their result are in the setting of Morrey spaces over metric measure space.

Theorem 4.2. [15] Let $\theta \in(0,1), 1 \leq q_{0} \leq p_{0}<\infty$, and $1 \leq q_{1} \leq p_{1}<\infty$. Assume that $\frac{p_{0}}{q_{0}}=\frac{p_{1}}{q_{1}}$. Then

$$
\left[\mathcal{M}_{q_{0}}^{p_{0}}, \mathcal{M}_{q_{1}}^{p_{1}}\right]_{\theta}=\overline{\mathcal{M}_{q_{0}}^{p_{0}} \cap \mathcal{M}_{q_{1}}^{p_{1}}} \mathcal{M}_{q}^{p},
$$

where $p$ and $q$ are defined by (26).

The key parts of the proof of Theorem 4.2 are Lemma 3.15 and the calculation of the Calderón product between $\mathcal{M}_{q_{0}}^{p_{0}}$ and $\mathcal{M}_{q_{1}}^{p_{1}}$.

Proposition 4.3. Let $\theta \in(0,1), 1 \leq q_{0} \leq p_{0}<\infty$, and $1 \leq q_{1} \leq p_{1}<\infty$. Assume that $\frac{p_{0}}{q_{0}}=\frac{p_{1}}{q_{1}}$. Define $p$ and $q$ by (26). Then

$$
\left(\mathcal{M}_{q_{0}}^{p_{0}}\right)^{1-\theta}\left(\mathcal{M}_{q_{1}}^{p_{1}}\right)^{\theta}=\mathcal{M}_{q}^{p} .
$$

Proof. Let $B=B(a, r)$ be any ball in $\mathbb{R}^{n}$ and $\varepsilon>0$. Let $f \in\left(\mathcal{M}_{q_{0}}^{p_{0}}\right)^{1-\theta}\left(\mathcal{M}_{q_{1}}^{p_{1}}\right)^{\theta}$. Then, there exist some functions $f_{0} \in \mathcal{M}_{q_{0}}^{p_{0}}$ and $f_{1} \in \mathcal{M}_{q_{1}}^{p_{1}}$ such that

$$
|f(x)| \leq\left|f_{0}(x)\right|^{1-\theta}\left|f_{1}(x)\right|^{\theta} \text {, a.e. } x \in \mathbb{R}^{n}
$$

and

$$
\left\|f_{0}\right\|_{\mathcal{M}_{q_{0}}^{p_{0}}}^{1-\theta}\left\|f_{1}\right\|_{\mathcal{M}_{q_{1}}^{p_{1}}}^{\theta} \leq(1+\varepsilon)\|f\|_{\left(\mathcal{M}_{q_{0}}^{p_{0}}\right)^{1-\theta}\left(\mathcal{M}_{q_{1}}^{p_{1}}\right)^{\theta}} .
$$

By using Hölder's inequality and (30), we have

$$
\begin{aligned}
\left(\int_{B}|f(x)|^{q} d x\right)^{\frac{1}{q}} & \leq\left(\int_{B}\left|f_{0}(x)\right|^{q(1-\theta)}\left|f_{1}(x)\right|^{q \theta} d x\right)^{\frac{1}{q}} \\
& \leq\left(\int_{B}\left|f_{0}(x)\right|^{q_{0}} d x\right)^{\frac{1-\theta}{q_{0}}}\left(\int_{B}\left|f_{1}(x)\right|^{q_{1}} d x\right)^{\frac{\theta}{q_{1}}} .
\end{aligned}
$$

Combining $\frac{p_{0}}{q_{0}}=\frac{p_{1}}{q_{1}},(26)$, and inequalities (31)-(32), we obtain

$$
\begin{aligned}
|B|^{\frac{1}{p}-\frac{1}{q}}\left(\int_{B}|f(x)|^{q} d x\right)^{\frac{1}{q}} & \leq \frac{|B|^{\frac{1-\theta}{p_{0}}+\frac{\theta}{p_{1}}}}{|B|^{\frac{1-\theta}{q_{0}}+\frac{\theta}{q_{1}}}}\left\|f_{0}\right\|_{L^{q_{0}}(B)}^{1-\theta}\left\|f_{1}\right\|_{L^{q_{1}}(B)}^{\theta} \\
& \leq\left\|f_{0}\right\|_{\mathcal{M}_{q_{0}}^{p_{0}}}^{1-\theta}\left\|f_{1}\right\|_{\mathcal{M}_{q_{1}}^{p_{1}}}^{\theta} \\
& \leq(1+\varepsilon)\|f\|_{\left(\mathcal{M}_{q_{0}}^{p_{0}}\right)^{1-\theta}\left(\mathcal{M}_{q_{1}}^{p_{1}}\right)^{\theta} .}
\end{aligned}
$$


Since $\varepsilon$ is arbitary, we have $f \in \mathcal{M}_{q}^{p}$ with $\|f\|_{\mathcal{M}_{q}^{p}} \leq\|f\|_{\left(\mathcal{M}_{q_{0}}^{p_{0}}\right)^{1-\theta}\left(\mathcal{M}_{q_{1}}^{p_{1}}\right)^{\theta}}$. Thus,

$$
\left(\mathcal{M}_{q_{0}}^{p_{0}}\right)^{1-\theta}\left(\mathcal{M}_{q_{1}}^{p_{1}}\right)^{\theta} \subseteq \mathcal{M}_{q}^{p} .
$$

Conversely, let $f \in \mathcal{M}_{q}^{p}$. Define $\tilde{f}_{j}:=|f|^{\frac{q}{q_{j}}}$ where $j \in\{0,1\}$. It follows from (26) and $\frac{p_{0}}{q_{0}}=\frac{p_{1}}{q_{1}}$ that

$$
\frac{p}{q}=\frac{p_{0}}{q_{0}}=\frac{p_{1}}{q_{1}} .
$$

Then $\tilde{f}_{j} \in \mathcal{M}_{q_{j}}^{p_{j}}$ with $\left\|\tilde{f}_{j}\right\|_{\mathcal{M}_{q_{j}}^{p_{j}}}=\|f\|_{\mathcal{M}_{q}^{p}}^{\frac{q}{q_{j}}}$ for $j=0,1$. Observe that, we have

$$
\left|\tilde{f}_{0}\right|^{1-\theta}\left|\tilde{f}_{1}\right|^{\theta}=|f|^{\frac{q(1-\theta)}{q_{0}}}|f|^{\frac{q \theta}{q_{1}}}=|f|
$$

and

$$
\|f\|_{\left(\mathcal{M}_{q_{0}}^{p_{0}}\right)^{1-\theta}\left(\mathcal{M}_{q_{1}}^{p_{1}}\right)^{\theta}} \leq\left\|\tilde{f}_{0}\right\|_{\mathcal{M}_{q_{0}}^{p_{0}}}^{1-\theta}\left\|\tilde{f}_{1}\right\|_{\mathcal{M}_{q_{1}}^{p_{1}}}^{\theta}=\|f\|_{\mathcal{M}_{q}^{p}}^{\frac{q(1-\theta)}{q_{0}}+\frac{q \theta}{q_{1}}}=\|f\|_{\mathcal{M}_{q}^{p}}<\infty .
$$

Consequently, $f \in\left(\mathcal{M}_{q_{0}}^{p_{0}}\right)^{1-\theta}\left(\mathcal{M}_{q_{1}}^{p_{1}}\right)^{\theta}$. Therefore, $\mathcal{M}_{q}^{p} \subseteq\left(\mathcal{M}_{q_{0}}^{p_{0}}\right)^{1-\theta}\left(\mathcal{M}_{q_{1}}^{p_{1}}\right)^{\theta}$. Thus, we have proved (29).

The description of the right-hand side of (28) and can be refined as follows.

Theorem 4.4. [10] Keep the same assumption as in Theorem 4.2 and assume also that $q_{0} \neq q_{1}$. Then we have

$$
\left[\mathcal{M}_{q_{0}}^{p_{0}}, \mathcal{M}_{q_{1}}^{p_{1}}\right]_{\theta}=\left\{f \in \mathcal{M}_{q}^{p}: \lim _{N \rightarrow \infty}\left\|f-\chi_{\left\{\frac{1}{N} \leq|f| \leq N\right\}} f\right\|_{\mathcal{M}_{q}^{p}}=0\right\} .
$$

Note that Theorem 4.4 is an improvement of Theorems 4.2, in the sense that, $\left[\mathcal{M}_{q_{0}}^{p_{0}}, \mathcal{M}_{q_{1}}^{p_{1}}\right]_{\theta}$ is now written in terms of the parameters $p$ and $q$ only and this description is more explicit than the right-hand side of (28). In order to prove Theorem 4.4, we need two lemmas. The first one is the fact that the set in the right-hand side of (36) is closed. The second lemma tells us that this set contains $\mathcal{M}_{q_{0}}^{p_{0}} \cap \mathcal{M}_{q_{1}}^{p_{1}}$.

Lemma 4.5. Let $1 \leq p \leq q<\infty$. Then the set

$$
A:=\left\{f \in \mathcal{M}_{q}^{p}: \lim _{N \rightarrow \infty}\left\|f-\chi_{\left\{\frac{1}{N} \leq|f| \leq N\right\}} f\right\|_{\mathcal{M}_{q}^{p}}=0\right\}
$$

is a closed subset $\mathcal{M}_{q}^{p}$.

Proof. Let $\left\{f_{j}\right\}_{j=1}^{\infty} \subset A$ be such that $f_{j}$ converges to $f$ in $\mathcal{M}_{q}^{p}$. Fix $j \in \mathbb{N}$. For every $N \in \mathbb{N}$, we have

$$
\left\|\chi_{\left\{|f|<\frac{1}{N}\right\}} f\right\|_{\mathcal{M}_{q}^{p}} \leq\left\|f-f_{j}\right\|_{\mathcal{M}_{q}^{p}}+\left\|\chi_{\left\{|f|<\frac{1}{N}\right\} \cap\left\{\left|f_{j}\right| \geq \frac{2}{N}\right\}} f_{j}\right\|_{\mathcal{M}_{q}^{p}}+\left\|\chi_{\left\{\left|f_{j}\right|<\frac{2}{N}\right\}} f_{j}\right\|_{\mathcal{M}_{q}^{p}}
$$

and

$$
\left\|\chi_{\{|f|>N\}} f\right\|_{\mathcal{M}_{q}^{p}} \leq\left\|f-f_{j}\right\|_{\mathcal{M}_{q}^{p}}+\left\|\chi_{\{|f|>N\} \cap\left\{\left|f_{j}\right| \leq \frac{N}{2}\right\}} f_{j}\right\|_{\mathcal{M}_{q}^{p}}+\left\|\chi_{\left\{\left|f_{j}\right|>\frac{N}{2}\right\}} f_{j}\right\|_{\mathcal{M}_{q}^{p}} .
$$


On the set $\left\{|f|<\frac{1}{N}\right\} \cap\left\{\left|f_{j}\right| \geq \frac{2}{N}\right\}$, we have

$$
\left|f_{j}\right| \leq\left|f_{j}-f\right|+|f|<\left|f_{j}-f\right|+\frac{1}{N} \leq\left|f_{j}-f\right|+\frac{1}{2}\left|f_{j}\right|,
$$

and hence $\left|f_{j}\right| \leq 2\left|f-f_{j}\right|$. Consequently,

$$
\left\|\chi_{\left\{|f|<\frac{1}{N}\right\}} f\right\|_{\mathcal{M}_{q}^{p}} \leq 3\left\|f-f_{j}\right\|_{\mathcal{M}_{q}^{p}}+\left\|\chi_{\left\{\left|f_{j}\right|<\frac{2}{N}\right\}} f_{j}\right\|_{\mathcal{M}_{q}^{p}} .
$$

Meanwhile, on the set $\{|f|>N\} \cap\left\{\left|f_{j}\right| \leq \frac{N}{2}\right\}$, we have

$$
\left|f_{j}\right| \leq \frac{N}{2}<\frac{|f|}{2} \leq \frac{\left|f-f_{j}\right|}{2}+\frac{\left|f_{j}\right|}{2},
$$

and hence, $\left|f_{j}\right| \leq\left|f-f_{j}\right|$. Therefore,

$$
\left\|\chi_{\{|f|>N\}} f\right\|_{\mathcal{M}_{q}^{p}} \leq 2\left\|f-f_{j}\right\|_{\mathcal{M}_{q}^{p}}+\left\|\chi_{\left\{\left|f_{j}\right|>\frac{N}{2}\right\}} f_{j}\right\|_{\mathcal{M}_{q}^{p}} .
$$

By combining (38) and (39), we get

$$
\begin{aligned}
\left\|f-\chi_{\left\{\frac{1}{N} \leq|f| \leq N\right\}} f\right\|_{\mathcal{M}_{q}^{p}} & \leq\left\|\chi_{\left\{|f|<\frac{1}{N}\right\}} f\right\|_{\mathcal{M}_{q}^{p}}+\left\|\chi_{\{|f|>N\}} f\right\|_{\mathcal{M}_{q}^{p}} \\
& \leq 5\left\|f-f_{j}\right\|_{\mathcal{M}_{q}^{p}}+\left\|\chi_{\left\{\left|f_{j}\right|<\frac{2}{N}\right\}} f_{j}\right\|_{\mathcal{M}_{q}^{p}}+\left\|\chi_{\left\{\left|f_{j}\right|>\frac{N}{2}\right\}} f_{j}\right\|_{\mathcal{M}_{q}^{p}} .
\end{aligned}
$$

Since $f_{j} \in A$, we have

$$
\limsup _{N \rightarrow \infty}\left\|f-\chi_{\left\{\frac{1}{N} \leq|f| \leq N\right\}} f\right\|_{\mathcal{M}_{q}^{p}} \leq 5\left\|f-f_{j}\right\|_{\mathcal{M}_{q}^{p}} .
$$

By taking $j \rightarrow \infty$, we have $\lim _{N \rightarrow \infty}\left\|f-\chi_{\left\{\frac{1}{N} \leq|f| \leq N\right\}} f\right\|_{\mathcal{M}_{q}^{p}}=0$, and hence, $f \in A$.

Lemma 4.6. [10] Maintain the same conditions as Proposition 4.3 and let $A$ be defined by (37). Then

$$
\mathcal{M}_{q_{0}}^{p_{0}} \cap \mathcal{M}_{q_{1}}^{p_{1}} \subseteq A .
$$

Proof. Without loss of generality, we assume that $q_{1}>q_{0}$. Then, $q_{1}>q>q_{0}$. Consequently, for every $f \in \mathcal{M}_{q_{0}}^{p_{0}} \cap \mathcal{M}_{q_{1}}^{p_{1}}$, we have

$$
\begin{aligned}
\left\|f-\chi_{\left\{\frac{1}{N} \leq|f| \leq N\right\}} f\right\|_{\mathcal{M}_{q}^{p}} & \leq\left\|\chi_{\left\{|f|<\frac{1}{N}\right\}}|f|^{1-\frac{q_{0}}{q}}|f|^{\frac{q_{0}}{q}}\right\|_{\mathcal{M}_{q}^{p}}+\left\|\chi_{\{|f|>N\}}|f|^{1-\frac{q_{1}}{q}}|f|^{\frac{q_{1}}{q}}\right\|_{\mathcal{M}_{q}^{p}} \\
& \leq N^{\frac{q_{0}-q}{q}}\left\||f|^{\frac{q_{0}}{q}}\right\|_{\mathcal{M}_{q}^{p}}+N^{\frac{q-q_{1}}{q}}\left\||f|^{\frac{q_{1}}{q}}\right\|_{\mathcal{M}_{q}^{p}} \\
& =N^{\frac{q_{0}-q}{q}}\|f\|_{\mathcal{M}_{q_{0}}^{p_{0}}}^{\frac{q_{0}}{q}}+N^{\frac{q-q_{1}}{q}}\|f\|_{\mathcal{M}_{q_{1}}^{p_{1}}}^{\frac{q_{1}}{p_{1}}} \rightarrow 0
\end{aligned}
$$

as $N \rightarrow \infty$, which implies $f \in A$.

We are now ready to prove Theorem 4.4.

Proof of Theorem 4.4. By virtue of Theorem 4.2 and Lemmas 4.5 and 4.6, we have

$$
\left[\mathcal{M}_{q_{0}}^{p_{0}}, \mathcal{M}_{q_{1}}^{p_{1}}\right]_{\theta}=\overline{\mathcal{M}_{q_{0}}^{p_{0}} \cap \mathcal{M}_{q_{1}}^{p_{1}} \mathcal{M}_{q}^{p}} \subseteq A .
$$


Conversely, let $f \in A$. For every $N \in \mathbb{N}$, define $f_{N}:=\chi_{\left\{\frac{1}{N} \leq|f| \leq N\right\}} f$. As in the proof of Lemma 4.6, we may assume that $q_{0}<q_{1}$. Then $q_{0}<q<q_{1}$. This implies

$$
\left\|f_{N}\right\|_{\mathcal{M}_{q_{0}}^{p_{0}}} \leq\left\|\chi_{\left\{\frac{1}{N} \leq|f|\right\}}|f|^{1-\frac{q}{q_{0}}}|f|^{\frac{q}{q_{0}}}\right\|_{\mathcal{M}_{q_{0}}^{p_{0}}} \leq N^{\frac{q-q_{0}}{q_{0}}}\|f\|_{\mathcal{M}_{q}^{p}}^{q / q_{0}}<\infty
$$

and

$$
\left\|f_{N}\right\|_{\mathcal{M}_{q_{1}}^{p_{1}}} \leq\left\|\chi_{\{|f|<N\}}|f|^{1-\frac{q}{q_{1}}}|f|^{\frac{q}{q_{1}}}\right\|_{\mathcal{M}_{q_{1}}^{p_{1}}} \leq N^{\frac{q_{1}-q}{q_{1}}}\|f\|_{\mathcal{M}_{q}^{p}}^{q / q_{1}}<\infty .
$$

Therefore, $f \in \overline{\mathcal{M}_{q_{0}}^{p_{0}} \cap \mathcal{M}_{q_{1}}^{p_{1}}} \mathcal{M}_{q}^{p}$ by the definition of $A$. According to Theorem 4.2, we have $f \in\left[\mathcal{M}_{q_{0}}^{p_{0}}, \mathcal{M}_{q_{1}}^{p_{1}}\right]_{\theta}$ as desired.

4.2. The second complex interpolation of Morrey spaces. Observe that the function $f(x):=|x|^{-n / p}$ does not belong to the set in the right-hand side of (36), but this function is in $\mathcal{M}_{q}^{p}$. From this observation, one may inquire whether we can interpolate Morrey spaces and that the output is also Morrey spaces. The affirmative answer was given by Lemarié-Rieusset [14]. He proved the following result about the second complex interpolation of Morrey spaces.

Theorem 4.7. [14] Keep the same asssumption as in Theorem 4.2. Then

$$
\left[\mathcal{M}_{q_{0}}^{p_{0}}, \mathcal{M}_{q_{1}}^{p_{1}}\right]^{\theta}=\mathcal{M}_{q}^{p} .
$$

It is written in the book $[1$, p. 90$]$ that the first complex interpolation space is the main interest in this book and the second complex interpolation method is considered as a technical tool. Therefore, Theorem 4.7 can be seen as an example of the importance of the second complex interpolation method.

In order to prove Theorem 4.7, we prove the following lemmas about the construction of the second complex interpolation functor.

Lemma 4.8. [9, Lemma 4] Let $q_{0}>q_{1}$ and $f \in L^{0}$. Define $q: \bar{S} \rightarrow \mathbb{C}, F: \bar{S} \rightarrow L^{0}$ and $G: \bar{S} \rightarrow L^{0}$ by:

$$
\begin{gathered}
\frac{1}{q(z)}=\frac{1-z}{q_{0}}+\frac{z}{q_{1}}, \\
F(z):=\operatorname{sgn}(f) \exp \left(\frac{q}{q(z)} \log |f|\right) \quad(z \in \bar{S}),
\end{gathered}
$$

and

$$
G(z):=(z-\theta) \int_{0}^{1} F(\theta+(z-\theta) t) d t \quad(z \in \bar{S}),
$$

respectively. Define $F_{0}, F_{1}, G_{0}, G_{1}: \bar{S} \rightarrow L^{0}$ by:

$$
F_{0}(z):=F(z) \chi_{\{|f| \leq 1\}}, \quad F_{1}(z):=F(z) \chi_{\{|f|>1\}},
$$

and

$$
G_{0}(z):=G(z) \chi_{\{|f| \leq 1\}}, \quad G_{1}(z):=G(z) \chi_{\{|f|>1\}} .
$$

Then, for any $z \in \bar{S}$, we have

$$
|G(z)| \leq(1+|z|)\left(|f|^{q / q_{0}}+|f|^{q / q_{1}}\right) .
$$


For any $z \in \mathbb{C}$ with $\varepsilon<\operatorname{Re}(z)<1-\varepsilon$ and $w \in \mathbb{C}$ with $|w| \ll 1$, we have

$$
\begin{aligned}
& \left|\frac{G_{0}(z+w)-G_{0}(z)}{w}-F_{0}(z)\right| \leq C_{\varepsilon}|w| \cdot|f|^{\frac{q}{q_{0}}}, \\
& \left|\frac{G_{1}(z+w)-G_{1}(z)}{w}-F_{1}(z)\right| \leq C_{\varepsilon}|w| \cdot|f|^{\frac{q}{q_{1}}},
\end{aligned}
$$

where the constant $C_{\varepsilon}$ depending only on $\varepsilon \in(0,1 / 2)$.

Proof. For $t \in[0,1]$, define $v:=(z-\theta) t+\theta$. Since $\operatorname{Re}(v) \in[0,1]$, we have

$$
\begin{aligned}
|F(v)| & \leq|f|^{\frac{q}{q_{0}}(1-\operatorname{Re}(v))+\frac{q}{q_{1}} \operatorname{Re}(v)} \\
& \leq(1-\operatorname{Re}(v))|f|^{\frac{q}{q_{0}}}+\operatorname{Re}(v)|f|^{\frac{q}{q_{1}}} \leq|f|^{\frac{q}{q_{0}}}+|f|^{\frac{q}{q_{1}}} .
\end{aligned}
$$

By the triangle inequality, we have

$$
|G(z)| \leq|z-\theta|\left(|f|^{\frac{q}{q_{0}}}+|f|^{\frac{q}{q_{1}}}\right) \leq(1+|z|)\left(|f|^{\frac{q}{q_{0}}}+|f|^{\frac{q}{q_{1}}}\right)
$$

Writing out the definitions in full, we obtain

$$
\begin{aligned}
& \left|\frac{G_{0}(z+w)-G_{0}(z)}{w}-F_{0}(z)\right| \\
& =\left|F_{0}(\operatorname{Re}(z))\right|\left|\frac{\exp \left[q\left(\frac{-w}{q_{0}}+\frac{w}{q_{1}}\right) \log |f|\right]-1}{w\left(\frac{q}{q_{1}}-\frac{q}{q_{0}}\right) \log |f|}-1\right| .
\end{aligned}
$$

Since $q_{0}>q_{1}$, we have

$$
\begin{aligned}
& \left|\frac{G_{0}(z+w)-G_{0}(z)}{w}-F_{0}(z)\right| \\
& =\chi_{\{|f| \leq 1\}}|f|^{\frac{q}{q_{0}}(1-\operatorname{Re}(z))+\frac{q}{q_{1}} \operatorname{Re}(z)}\left|\frac{\exp \left[q\left(\frac{-w}{q_{0}}+\frac{w}{q_{1}}\right) \log |f|\right]-1}{w\left(\frac{q}{q_{1}}-\frac{q}{q_{0}}\right) \log |f|}-1\right| \\
& \leq \chi_{\{|f| \leq 1\}}|f|^{\frac{q}{q_{0}}} \cdot|f|^{\left(\frac{q}{q_{1}}-\frac{q}{q_{0}}\right) \varepsilon}\left|\frac{\exp \left[q\left(\frac{-w}{q_{0}}+\frac{w}{q_{1}}\right) \log |f|\right]-1}{w\left(\frac{q}{q_{1}}-\frac{q}{q_{0}}\right) \log |f|}-1\right| \\
& \leq|f|^{\frac{q}{q_{0}}} \sup _{0<t \leq 1} t\left(\frac{q}{q_{1}}-\frac{q}{q_{0}}\right) \varepsilon\left|\frac{\exp \left[q\left(\frac{-w}{q_{0}}+\frac{w}{q_{1}}\right) \log t\right]-1}{w\left(\frac{q}{q_{1}}-\frac{q}{q_{0}}\right) \log t}-1\right| \leq C_{\varepsilon}|w| \cdot|f|^{\frac{q}{q_{0}}} .
\end{aligned}
$$


By a similar argument, we also have

$$
\begin{aligned}
& \left|\frac{G_{1}(z+w)-G_{1}(z)}{w}-F_{1}(z)\right| \\
& =\chi_{\{|f|>1\}}|f|^{\frac{q}{q_{1}}} \cdot|f|^{\left(\frac{q}{q_{0}}-\frac{q}{q_{1}}\right)(1-\operatorname{Re}(z))}\left|\frac{\exp \left[q\left(\frac{-w}{q_{0}}+\frac{w}{q_{1}}\right) \log |f|\right]-1}{w\left(\frac{q}{q_{1}}-\frac{q}{q_{0}}\right) \log |f|}-1\right|
\end{aligned}
$$

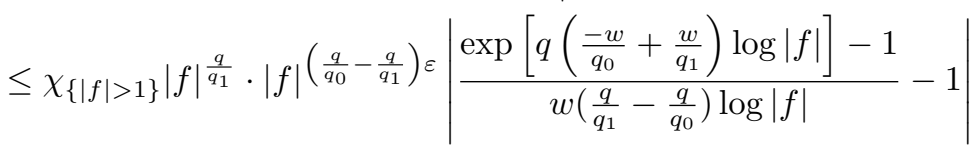

$$
\begin{aligned}
& \leq|f|^{\frac{q}{q_{1}}} \sup _{t \geq 1} t^{\left(\frac{q}{q_{0}}-\frac{q}{q_{1}}\right) \varepsilon}\left|\frac{\exp \left[q\left(\frac{-w}{q_{0}}+\frac{w}{q_{1}}\right) \log t\right]-1}{w\left(\frac{q}{q_{1}}-\frac{q}{q_{0}}\right) \log t}-1\right| \leq C_{\varepsilon}|w| \cdot|f|^{\frac{q}{q_{1}}}
\end{aligned}
$$

as desired.

Lemma 4.9. $\left[9\right.$, Lemma 12] Let $f \in \mathcal{M}_{q}^{p}$. Via (40) define $F: \bar{S} \rightarrow \mathcal{M}_{q_{0}}^{p_{0}}+\mathcal{M}_{q_{1}}^{p_{1}}$ and $G: \bar{S} \rightarrow \mathcal{M}_{q_{0}}^{p_{0}}+\mathcal{M}_{q_{1}}^{p_{1}}$ by (41) and (42), respectively. Then, the function $G$ belongs to $\mathcal{G}\left(\mathcal{M}_{q_{0}}^{p_{0}}, \mathcal{M}_{q_{1}}^{p_{1}}\right)$.

Proof. It follows from (45) that $G(z) \in \mathcal{M}_{q_{0}}^{p_{0}}+\mathcal{M}_{q_{1}}^{p_{1}}$ and

$$
\sup _{z \in \bar{S}} \frac{\|G(z)\|_{\mathcal{M}_{q_{0}}^{p_{0}}+\mathcal{M}_{q_{1}}^{p_{1}}}}{1+|z|} \leq\|f\|_{\mathcal{M}_{q}^{p}}^{q / q_{0}}+\|f\|_{\mathcal{M}_{q}^{p}}^{q / q_{1}} .
$$

Now let $z_{1}, z_{2} \in \bar{S}$. Then, by inequality (48), we get

$$
\left\|G\left(z_{1}\right)-G\left(z_{2}\right)\right\|_{\mathcal{M}_{q_{0}}^{p_{0}}+\mathcal{M}_{q_{1}}^{p_{1}}} \leq\left|z_{1}-z_{2}\right|\left(\|f\|_{\mathcal{M}_{q}^{p}}^{q / q_{0}}+\|f\|_{\mathcal{M}_{q}^{p}}^{q / q_{1}}\right) .
$$

This shows the continuity of $G: \bar{S} \rightarrow \mathcal{M}_{q_{0}}^{p_{0}}+\mathcal{M}_{q_{1}}^{p_{1}}$. The proof of holomorphicity of $G: S \rightarrow \mathcal{M}_{q_{0}}^{p_{0}}+\mathcal{M}_{q_{1}}^{p_{1}}$ goes as follows. Let $\varepsilon \in\left(0, \frac{1}{2}\right)$ and define

$$
S_{\varepsilon}:=\{z \in S: \varepsilon<\operatorname{Re}(z)<1-\varepsilon\} .
$$

According to (46) and (47), we have

$$
\begin{aligned}
& \left\|\frac{G(z+w)-G(z)}{w}-F(z)\right\|_{\mathcal{M}_{q_{0}}^{p_{0}}+\mathcal{M}_{q_{1}}^{p_{1}}} \\
& \quad \leq\left\|\frac{G_{0}(z+w)-G_{0}(z)}{w}-F_{0}(z)\right\|_{\mathcal{M}_{q_{0}}^{p_{0}}}+\left\|\frac{G_{1}(z+w)-G_{1}(z)}{w}-F_{1}(z)\right\|_{\mathcal{M}_{q_{1}}^{p_{1}}} \\
& \quad \leq C_{\varepsilon}|w|\left(\|f\|_{\mathcal{M}_{q}^{p}}^{q / q_{0}}+\|f\|_{\mathcal{M}_{q}^{p}}^{q / q_{1}}\right) .
\end{aligned}
$$

Taking $w \rightarrow 0$, we see that $G: S_{\varepsilon} \rightarrow \mathcal{M}_{q_{0}}^{p_{0}}+\mathcal{M}_{q_{1}}^{p_{1}}$ is holomorphic. Since $\varepsilon>0$ is arbitrary, we conclude that $G: S \rightarrow \mathcal{M}_{q_{0}}^{p_{0}}+\mathcal{M}_{q_{1}}^{p_{1}}$ is holomorphic.

We now verify that $G\left(j+i t_{1}\right)-G\left(j+i t_{2}\right) \in \mathcal{M}_{q_{j}}^{p_{j}}$ for every $t_{1}, t_{2} \in \mathbb{R}$ and $j \in\{0,1\}$ and also

$$
\|G(j+i \cdot)\|_{\operatorname{Lip}\left(\mathbb{R}, \mathcal{M}_{q_{j}}^{p_{j}}\right)} \leq\left(\|f\|_{\mathcal{M}_{q}^{p}}\right)^{q / q_{j}}
$$


for every $j \in\{0,1\}$. Combining $|F(j+i t)|=|f|^{\frac{q}{q_{j}}}$ and

$$
G\left(j+i t_{1}\right)-G\left(j+i t_{2}\right)=-i \int_{t_{1}}^{t_{2}} F(j+i t) d t,
$$

we get

$$
\left\|G\left(j+i t_{1}\right)-G\left(j+i t_{2}\right)\right\|_{\mathcal{M}_{q_{j}}^{p_{j}}} \leq\left|t_{1}-t_{2}\right|\|f\|_{\mathcal{M}_{q}^{p}}^{\frac{q}{q_{j}}}
$$

This implies (50). Thus, $G \in \mathcal{G}\left(\mathcal{M}_{q_{0}}^{p_{0}}, \mathcal{M}_{q_{1}}^{p_{1}}\right)$ with

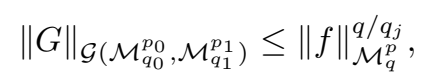

as desired.

Note that we can not use the function $F$ defined by (41) as the first complex interpolation functor because $F$ does not belong to $\mathcal{F}\left(\mathcal{M}_{q_{0}}^{p_{0}}, \mathcal{M}_{q_{1}}^{p_{1}}\right)$ when $f(x):=$ $|x|^{-n / p}$. This fact is a consequence of the following proposition.

Proposition 4.10. [9, Proposition 4] Let $f(x):=|x|^{-n / p}$ and define $F$ by (41). Then the mapping $t \in \mathbb{R} \mapsto F(i t) \in \mathcal{M}_{q_{0}}^{p_{0}}$ is not continuous at $t=0$.

Proof. Assume that $p_{0}>p_{1}$ and define $Q:=\frac{1}{p_{1}}-\frac{1}{p_{0}}$. Using $\frac{p_{0}}{q_{0}}=\frac{p}{q}=\frac{p_{1}}{q_{1}}$, for every $0<t<\frac{1}{Q}$, we have

$$
|F(i t)-F(0)|=\left.|x|^{-\frac{n}{p_{0}}}|| x\right|^{-Q i t}-\left.1|=2| x\right|^{-\frac{n}{p_{0}}}\left|\sin \left(\frac{Q t \log |x|}{2}\right)\right| .
$$

Using (51) and letting $R:=\exp \left((Q t)^{-1}\right)$, we get

$$
\begin{aligned}
& \|F(i t)-F(0)\|_{\mathcal{M}_{q_{0}}^{p_{0}}} \\
& \geq 2|B(0,2 R)|^{\frac{1}{p_{0}}-\frac{1}{q_{0}}}\left(\int_{B(0,2 R) \backslash B(0, R)}|x|^{-\frac{n q_{0}}{p_{0}}}\left|\sin \left(\frac{Q t \log |x|}{2}\right)\right|^{q_{0}} d x\right)^{\frac{1}{q_{0}}} \\
& \gtrsim R^{\frac{n}{p_{0}}-\frac{n}{q_{0}}}\left(\int_{B(0,2 R) \backslash B(0, R)}|x|^{-\frac{n q_{0}}{p_{0}}} d x\right)^{\frac{1}{q_{0}}} \gtrsim 1,
\end{aligned}
$$

where we use

$$
\left|\sin \left(\frac{Q t \log |x|}{2}\right)\right|>\sin \left(\frac{1}{2}\right)
$$

for every $R<|x|<2 R$. Thus, (52) implies

$$
\lim _{t \rightarrow 0^{+}}\|F(i t)-F(0)\|_{\mathcal{M}_{q_{0}}^{p_{0}}} \neq 0
$$

as desired.

Now we arrive at our main result in this section.

Theorem 4.11. [9, p. 316] Keep the same assumption as in Proposition 4.3. Then

$$
\left[\mathcal{M}_{q_{0}}^{p_{0}}, \mathcal{M}_{q_{1}}^{p_{1}}\right]^{\theta}=\mathcal{M}_{q}^{p} .
$$


Proof. Let $f \in \mathcal{M}_{q}^{p}$. By a normalization, we may suppose $\|f\|_{\mathcal{M}_{q}^{p}}=1$, for the purpose of proving $f \in\left[\mathcal{M}_{q_{0}}^{p_{0}}, \mathcal{M}_{q_{1}}^{p_{1}}\right]^{\theta}$. For every $z \in \bar{S}$, define $F(z)$ and $G(z)$ as we did in Lemma 4.8. Thanks to Lemma 4.9, we have $G \in \mathcal{G}\left(\mathcal{M}_{q_{0}}^{p_{0}}, \mathcal{M}_{q_{1}}^{p_{1}}\right)$. Since $G^{\prime}(\theta)=F(\theta)=f$, we have

$$
\|f\|_{\left[\mathcal{M}_{q_{0}}^{p_{0}}, \mathcal{M}_{q_{1}}^{p_{1}}\right]^{\theta}} \leq\|G\|_{\mathcal{G}\left(\mathcal{M}_{q_{0}}^{p_{0}}, \mathcal{M}_{q_{1}}^{p_{1}}\right)}=\max _{j=0,1}\|G(j+i \cdot)\|_{\operatorname{Lip}\left(\mathbb{R}, \mathcal{M}_{q_{j}}^{p_{j}}\right)}=1 .
$$

This shows that $\left[\mathcal{M}_{q_{0}}^{p_{0}}, \mathcal{M}_{q_{1}}^{p_{1}}\right]^{\theta} \supset \mathcal{M}_{q}^{p}$. Conversely, let $f \in\left[\mathcal{M}_{q_{0}}^{p_{0}}, \mathcal{M}_{q_{1}}^{p_{1}}\right]^{\theta}$ with

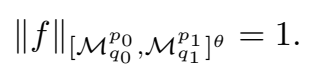

Suppose $f$ is realized as $G^{\prime}(\theta)$, where $G \in \mathcal{G}\left(\mathcal{M}_{q_{0}}^{p_{0}}, \mathcal{M}_{q_{1}}^{p_{1}}\right)$ and $\|G\|_{\mathcal{G}\left(\mathcal{M}_{q_{0}}^{p_{0}}, \mathcal{M}_{q_{1}}^{p_{1}}\right)} \leq 2$. For every $k \in \mathbb{N}$ and $z \in \bar{S}$, we define $H_{k}(z)$ by (22). According to Lemma 3.11 and Theorem 4.4, we obtain

$$
\left\|H_{k}(\theta)\right\|_{\mathcal{M}_{q}^{p}} \lesssim\left\|H_{k}(\theta)\right\|_{\left[\mathcal{M}_{q_{0}}^{p_{0}}, \mathcal{M}_{q_{1}}^{p_{1}}\right]_{\theta}} \leq\|G\|_{\mathcal{G}_{\left(\mathcal{M}_{q_{0}}, \mathcal{M}_{q_{1}}^{p_{1}}\right)} \leq 2 .}
$$

Meanwhile, since $f=G^{\prime}(\theta)=\lim _{k \rightarrow \infty} H_{k}(\theta)$ in $\mathcal{M}_{q_{0}}^{p_{0}}+\mathcal{M}_{q_{1}}^{p_{1}}$, there exists a subsequence $\left\{H_{k_{j}}\right\}_{j=1}^{\infty}$ such that $f(x)=\lim _{j \rightarrow \infty} H_{k_{j}}(\theta)(x)$ for almost every $x \in \mathbb{R}^{n}$. Consequently, by virtue of the Fatou lemma and (54), we have

$$
\|f\|_{\mathcal{M}_{q}^{p}} \lesssim \liminf _{j \rightarrow \infty}\left\|H_{k_{j}}(\theta)\right\|_{\mathcal{M}_{q}^{p}} \leq 2 .
$$

This implies $\left[\mathcal{M}_{q_{0}}^{p_{0}}, \mathcal{M}_{q_{1}}^{p_{1}}\right]^{\theta} \hookrightarrow \mathcal{M}_{q}^{p}$.

5. The Description of COMPlex interpolation of subspaces of Morrey SPACES

The first result on the complex interpolation of subspaces of Morrey spaces can be traced back to [20]. They investigated the space $\mathcal{M}_{q}^{p}:={\overline{C_{\mathrm{c}}^{\infty}}}^{\mathcal{M}_{q}^{p}}$, where $C_{\mathrm{c}}^{\infty}$ is the set of all infinitely differentiable functions with compact support. Their result is given as follows.

Theorem 5.1. Let $\theta \in(0,1), 1<q_{0} \leq p_{0}<\infty$, and $1<q_{1} \leq p_{1}<\infty$. Assume that $\frac{p_{0}}{q_{0}}=\frac{p_{1}}{q_{1}}$. Define $p$ and $q$ by

$$
\frac{1}{p}:=\frac{1-\theta}{p_{0}}+\frac{\theta}{p_{1}} \quad \text { and } \quad \frac{1}{q}:=\frac{1-\theta}{q_{0}}+\frac{\theta}{q_{1}} .
$$

Then

$$
\left[\stackrel{\circ}{\mathcal{M}}_{q_{0}}^{p_{0}}, \stackrel{\circ}{\mathcal{M}}_{q_{1}}^{p_{1}}\right]_{\theta}=\left[\mathcal{M}_{q_{0}}^{p_{0}}, \stackrel{\circ}{\mathcal{M}}_{q_{1}}^{p_{1}}\right]_{\theta}=\left[\stackrel{\circ}{\mathcal{M}}_{q_{0}}^{p_{0}}, \mathcal{M}_{q_{1}}^{p_{1}}\right]_{\theta}=\stackrel{\circ}{\mathcal{M}}_{q}^{p}
$$

Let $1 \leq q \leq p<\infty$. We now consider the following subspaces:

(1) $\overline{\mathcal{M}}_{q}^{p}:={\overline{L^{\infty} \cap \mathcal{M}_{q}^{p}}}^{\mathcal{M}_{q}^{p}}$ (see [5]).

(2) Denote by $L_{\mathrm{c}}^{0}$ the set of all measurable functions with compact support and define $\mathcal{M}_{q}^{*}:={\overline{L_{\mathrm{c}}^{0} \cap \mathcal{M}_{q}^{p}}}^{\mathcal{M}_{q}^{p}}$ (see [21]).

(3) $\widetilde{\mathcal{M}}_{q}^{p}:=\overline{L_{\mathrm{c}}^{\infty}} \mathcal{M}_{q}^{p}$ (see [9]). 
Observe that $\widetilde{\mathcal{M}_{q}^{p}}$ coincides with ${\overline{C_{\mathrm{c}}^{\infty}}}^{\mathcal{M}_{q}^{p}}$. These subspaces can be unified by introducing the following definition.

Definition 5.2. Assume that a linear subspace of measurable functions $U$ satisfies the condition: $g \in U$ whenever $f \in U$ and $|g| \leq|f|$. For $1 \leq q \leq p<\infty$, define

$$
U \mathcal{M}_{q}^{p}:={\overline{U \cap \mathcal{M}_{q}^{p}}}^{\mathcal{M}_{q}^{p}} \text {. }
$$

Example 5.3. If $U:=L_{\mathrm{c}}^{\infty}, L_{\mathrm{c}}^{0}, L^{\infty}$, then $U \mathcal{M}_{q}^{p}=\widetilde{\mathcal{M}_{q}^{p}}, \mathcal{M}_{q}^{p}, \overline{\mathcal{M}}_{q}^{p}$.

Theorem 5.4. Let $\theta \in(0,1), 1 \leq q_{0} \leq p_{0}<\infty, 1 \leq q_{1} \leq p_{1}<\infty, \frac{p_{0}}{q_{0}}=\frac{p_{1}}{q_{1}}$, and $q_{0} \neq q_{1}$. Define

Then

$$
\frac{1}{p}=\frac{1-\theta}{p_{0}}+\frac{\theta}{p_{1}} \quad \text { and } \quad \frac{1}{q}:=\frac{1-\theta}{q_{0}}+\frac{\theta}{q_{1}} .
$$

$$
\left[U \mathcal{M}_{q_{0}}^{p_{0}}, U \mathcal{M}_{q_{1}}^{p_{1}}\right]_{\theta}=\left\{f \in U \mathcal{M}_{q}^{p}: \lim _{N \rightarrow \infty}\left\|f-\chi_{\left\{\frac{1}{N} \leq|f| \leq N\right\}} f\right\|_{\mathcal{M}_{q}^{p}}=0\right\} .
$$

In order to prove Theorem 5.4, we need to prove the following lemmas:

Lemma 5.5. [9, Lemma 4.2] Assume the same paramaters as in Theorem 5.4. Let $E$ be a measurable set such that $\chi_{E} \in U \mathcal{M}_{q}^{p}$. Then

$$
\chi_{E} \in U \mathcal{M}_{q_{0}}^{p_{0}} \cap U \mathcal{M}_{q_{1}}^{p_{1}} .
$$

Proof. Let $\chi_{E} \in U \mathcal{M}_{q}^{p}$ and choose $\left\{g_{k}\right\}_{k=1}^{\infty} \subseteq U \cap \mathcal{M}_{q}^{p}$ for which

$$
\lim _{k \rightarrow \infty}\left\|\chi_{E}-g_{k}\right\|_{\mathcal{M}_{q}^{p}}=0 \text {. }
$$

Define $h_{k}:=\chi_{\left\{g_{k} \neq 0\right\} \cap E}$. Then, for each $k=0,1$, we have

$$
\left\|\chi_{E}-h_{k}\right\|_{\mathcal{M}_{q_{j}}^{p_{j}}}=\left\|\chi_{E}-h_{k}\right\|_{\mathcal{M}_{q}^{p}}^{q / q_{j}} \leq\left\|\chi_{E}-g_{k}\right\|_{\mathcal{M}_{q}^{p}}^{q / q_{j}} \rightarrow 0
$$

as $k \rightarrow \infty$. Thus, $\chi_{E} \in U \mathcal{M}_{q_{0}}^{p_{0}} \cap U \mathcal{M}_{q_{1}}^{p_{1}}$.

Lemma 5.6. [9, Lemma 4.1] Assume the same paramaters as in Theorem 5.4. Then $U \mathcal{M}_{q_{0}}^{p_{0}} \cap U \mathcal{M}_{q_{1}}^{p_{1}} \subseteq U \mathcal{M}_{q}^{p}$.

Proof. Without loss of generality assume that $q_{1}>q_{0}$. Let $f \in U \mathcal{M}_{q_{0}}^{p_{0}} \cap U \mathcal{M}_{q_{1}}^{p_{1}}$. In view of Lemma 4.6, we may assume $f=\chi_{\{1 / N \leq|f| \leq N\}} f$ for some $N \in \mathbb{N}$. By the lattice property of the spaces $U \mathcal{M}_{q_{0}}^{p_{0}}, U \mathcal{M}_{q_{1}}^{p_{1}}$ and $U \mathcal{M}_{q}^{p}$, we may assume $f=\chi_{E}$ for some measurable set $E$. Choose a sequence $\left\{g_{j}\right\}_{j=1}^{\infty} \subseteq U \cap \mathcal{M}_{q_{1}}^{p_{1}}$ such that

$$
\lim _{j \rightarrow \infty}\left\|f-g_{j}\right\|_{\mathcal{M}_{q_{1}}^{p_{1}}}=0 .
$$

Define $F_{j}:=\left\{g_{j} \neq 0\right\} \cap E$. Hence $\left|f-\chi_{F_{j}}\right| \leq 2$ and $\left|f-\chi_{F_{j}}\right| \leq\left|f-g_{j}\right|$. Consequently,

$$
\left\|f-\chi_{F_{j}}\right\|_{\mathcal{M}_{q}^{p}}=\left\|\left|f-\chi_{F_{j}}\right|^{1-\frac{q_{1}}{q}}\left|f-\chi_{F_{j}}\right|^{\frac{q_{1}}{q}}\right\|_{\mathcal{M}_{q}^{p}} \leq 2^{1-\frac{q_{1}}{q}}\left\|f-g_{j}\right\|_{\mathcal{M}_{q_{1}}^{p_{1}}}^{\frac{q_{1}}{p_{1}}} .
$$

This shows that $f \in U \mathcal{M}_{q}^{p}$.

The proof of Theorem 5.4 is given as follows: 
Proof of Theorem 5.4. We assume that $q_{1}>q_{0}$. By using Lemma 5.6, the inclusions

$$
\left[U \mathcal{M}_{q_{0}}^{p_{0}}, U \mathcal{M}_{q_{1}}^{p_{1}}\right]_{\theta} \subseteq\left[\mathcal{M}_{q_{0}}^{p_{0}}, \mathcal{M}_{q_{1}}^{p_{1}}\right]_{\theta} \subseteq \mathcal{M}_{q}^{p}
$$

and the fact that $X_{0} \cap X_{1}$ is a dense subset of $\left[X_{0}, X_{1}\right]_{\theta}$, we have $\left[U \mathcal{M}_{q_{0}}^{p_{0}}, U \mathcal{M}_{q_{1}}^{p_{1}}\right]_{\theta} \subseteq$ $U \mathcal{M}_{q}^{p}$. Consequently,

$$
\begin{aligned}
{\left[U \mathcal{M}_{q_{0}}^{p_{0}}, U \mathcal{M}_{q_{1}}^{p_{1}}\right]_{\theta} } & \subseteq U \mathcal{M}_{q}^{p} \cap\left[\mathcal{M}_{q_{0}}^{p_{0}}, \mathcal{M}_{q_{1}}^{p_{1}}\right]_{\theta} \\
& =\left\{f \in U \mathcal{M}_{q}^{p}: \lim _{N \rightarrow \infty}\left\|f-\chi_{\left\{\frac{1}{N} \leq|f| \leq N\right\}} f\right\|_{\left.\mathcal{M}_{q}^{p}=0\right\} .}\right.
\end{aligned}
$$

Conversely, let $f \in U \mathcal{M}_{q}^{p}$ be such that

$$
\lim _{N \rightarrow \infty}\left\|f-\chi_{\left\{\frac{1}{N} \leq|f| \leq N\right\}} f\right\|_{\mathcal{M}_{q}^{p}}=0
$$

Then, $f \in U \mathcal{M}_{q}^{p} \cap\left[\mathcal{M}_{q_{0}}^{p_{0}}, \mathcal{M}_{q_{1}}^{p_{1}}\right]_{\theta}$. Note that, for any $0<b<c<\infty$, we have a pointwise estimate:

$$
\chi_{\{b \leq|f| \leq c\}} \leq \frac{1}{b} \chi_{\{b \leq|f| \leq c\}}|f| \leq \frac{|f|}{b},
$$

so $\chi_{\{b \leq|f| \leq c\}} \in U \mathcal{M}_{q}^{p}$. From Lemma 5.5, it follows that $\chi_{\{b \leq|f| \leq c\}} \in U \mathcal{M}_{q_{0}}^{p_{0}} \cap$ $U \mathcal{M}_{q_{1}}^{p_{1}}$. For every $N \in \mathbb{N}$ and $z \in \bar{S}$, define

$$
F_{N}(z)=\operatorname{sgn}(f)|f|^{q\left(\frac{1-z}{q_{0}}+\frac{z}{q_{1}}\right)} \chi_{\left\{\frac{1}{N} \leq|f| \leq N\right\}} \cdot
$$

Decompose $F_{N}(z):=F_{N, 0}(z)+F_{N, 1}(z)$ where $F_{N, 0}(z):=F_{N}(z) \chi_{\{|f| \leq 1\}}$. Since

$$
\left|F_{N, 0}(z)\right| \leq \chi_{\left\{\frac{1}{N} \leq|f| \leq 1\right\}} \quad \text { and } \quad\left|F_{N, 1}(z)\right| \leq\left(N^{\frac{q}{q_{0}}}+N^{\frac{q}{q_{1}}}\right) \chi_{\{1 \leq|f| \leq N\}},
$$

we have $F_{N}(z)=F_{N, 0}(z)+F_{N, 1}(z) \in U \mathcal{M}_{q_{0}}^{p_{0}}+U \mathcal{M}_{q_{1}}^{p_{1}}$. Moreover, we also have $\sup _{z \in \bar{S}}\left\|F_{N}(z)\right\|_{U \mathcal{M}_{q_{0}}^{p_{0}}+U \mathcal{M}_{q_{1}}^{p_{1}}} \leq\left\|\chi_{\left\{\frac{1}{N} \leq|f| \leq 1\right\}}\right\|_{U \mathcal{M}_{q_{0}}^{p_{0}}}+\left(N^{\frac{q}{q_{0}}}+N^{\frac{q}{q_{1}}}\right)\left\|\chi_{\{1 \leq|f| \leq N\}}\right\|_{U \mathcal{M}_{q_{1}}^{p_{1}}}$.

Observe that for every $w \in \bar{S}$, we have

$$
\left|F_{N}^{\prime}(w)\right| \leq\left(\frac{q}{q_{0}}-\frac{q}{q_{1}}\right)\left(N^{\frac{q}{q_{0}}}+N^{\frac{q}{q_{1}}}\right)(\log N) \times \chi_{\left\{\frac{1}{N} \leq|f| \leq N\right\}} .
$$

Then we have

$$
\begin{aligned}
\| & F_{N}(z)-F_{N}\left(z^{\prime}\right) \|_{U \mathcal{M}_{q_{0}}^{p_{0}}+U \mathcal{M}_{q_{1}}^{p_{1}}} \\
= & \left\|\int_{z^{\prime}}^{z} F_{N}^{\prime}(w) d w\right\|_{U \mathcal{M}_{q_{0}}^{p_{0}}+U \mathcal{M}_{q_{1}}^{p_{1}}} \\
\leq & \left(\frac{q}{q_{0}}-\frac{q}{q_{1}}\right)\left(N^{\frac{q}{q_{0}}}+N^{\frac{q}{q_{1}}}\right)(\log N) \times\left(\left\|\chi_{\left\{\frac{1}{N} \leq|f| \leq N\right\}}\right\|_{U \mathcal{M}_{q_{0}}^{p_{0}}+U \mathcal{M}_{q_{1}}^{p_{1}}}\right)\left|z-z^{\prime}\right| \\
\leq & \left(\frac{q}{q_{0}}-\frac{q}{q_{1}}\right)\left(N^{\frac{q}{q_{0}}}+N^{\frac{q}{q_{1}}}\right) \log N \\
& \times\left(\left\|\chi_{\left\{\frac{1}{N} \leq|f| \leq 1\right\}}\right\|_{U \mathcal{M}_{q_{0}}^{p_{0}}}+\left\|\chi_{\{1<|f| \leq N\}}\right\|_{U \mathcal{M}_{q_{1}}^{p_{1}}}\right)\left|z-z^{\prime}\right|
\end{aligned}
$$


for all $z, z^{\prime} \in \bar{S}$. Thus, $F_{N}: \bar{S} \rightarrow U \mathcal{M}_{q_{0}}^{p_{0}}+U \mathcal{M}_{q_{1}}^{p_{1}}$ is a continuous function. Likewise we can check that $\left.F_{N}\right|_{S}: S \rightarrow U \mathcal{M}_{q_{0}}^{p_{0}}+U \mathcal{M}_{q_{1}}^{p_{1}}$ is a holomorphic function. Note that, for all $t \in \mathbb{R}$ and $j=0,1$, we have

$$
\left|F_{N}(j+i t)\right|=|f|^{\frac{q}{q_{j}}} \chi_{\left\{\frac{1}{N} \leq|f| \leq N\right\}} \leq N^{\frac{q}{q_{j}}} \chi_{\left\{\frac{1}{N} \leq|f| \leq N\right\}}
$$

so, $F_{N}(j+i t) \in U \mathcal{M}_{q_{j}}^{p_{j}}$. Furthermore, by using (56), we get

$$
\begin{aligned}
\left\|F_{N}(j+i t)-F_{N}\left(j+i t^{\prime}\right)\right\|_{U \mathcal{M}_{q_{j}}^{p_{j}}}= & \left\|\int_{j+i t^{\prime}}^{j+i t} F_{N}^{\prime}(w) d w\right\|_{U \mathcal{M}_{q_{j}}^{p_{j}}} \\
\leq & \left(\frac{q}{q_{0}}-\frac{q}{q_{1}}\right)\left(N^{\frac{q}{q_{0}}}+N^{\frac{q}{q_{1}}}\right) \log N \\
& \times\left\|\chi_{\{1 / N \leq|f| \leq N\}}\right\|_{U \mathcal{M}_{q_{j}}^{p_{j}}\left|t-t^{\prime}\right|}
\end{aligned}
$$

for all $t, t^{\prime} \in \mathbb{R}$. This shows that $t \in \mathbb{R} \mapsto F_{N}(j+i t) \in U \mathcal{M}_{q_{j}}^{p_{j}}$ are continuous functions. In total, we have showed that $F_{N} \in \mathcal{F}\left(U \mathcal{M}_{q_{0}}^{p_{0}}, U \mathcal{M}_{q_{1}}^{p_{1}}\right)$. Note that, for $M, N \in \mathbb{N}$ with $N<M$, we have

$$
\begin{aligned}
\left\|F_{M}(\theta)-F_{N}(\theta)\right\|_{\left[U \mathcal{M}_{q_{0}}^{p_{0}}, U \mathcal{M}_{q_{1}}^{p_{1}}\right]_{\theta}} & \leq\left\|F_{M}-F_{N}\right\|_{\mathcal{F}\left(U \mathcal{M}_{q_{0}}^{p_{0}}, U \mathcal{M}_{q_{1}}^{p_{1}}\right)} \\
& =\max _{j=0,1} \sup _{t \in \mathbb{R}}\left\|F_{M}(j+i t)-F_{N}(j+i t)\right\|_{U \mathcal{M}_{q_{j}}^{p_{j}}} \\
& =\max _{j=0,1} \sup _{t \in \mathbb{R}}\left\||f|^{q / q_{j}} \chi_{\left\{\frac{1}{M} \leq|f| \leq \frac{1}{N}\right\} \cup\{N \leq|f| \leq M\}}\right\|_{\mathcal{M}_{q_{j}}^{p_{j}}} \\
& =\max _{j=0,1}\left\|f \chi_{\left\{\frac{1}{M} \leq|f| \leq \frac{1}{N}\right\} \cup\{N \leq|f| \leq M\}}\right\|_{\mathcal{M}_{q}^{p}}^{q / q_{j}} \\
& \leq \max _{j=0,1}\left\|f-\chi_{\left\{\frac{1}{N} \leq|f| \leq N\right\}} f\right\|_{\mathcal{M}_{q}^{p}}^{q / q_{j}} .
\end{aligned}
$$

Since $\lim _{N \rightarrow \infty}\left\|f-\chi_{\left\{\frac{1}{N} \leq|f| \leq N\right\}} f\right\|_{\mathcal{M}_{q}^{p}}=0$, we see that

$$
\left\|F_{M}(\theta)-F_{N}(\theta)\right\|_{\left[U \mathcal{M}_{q_{0}}^{p_{0}}, U \mathcal{M}_{q_{1}}^{p_{1}}\right]_{\theta}} \rightarrow 0
$$

whenever $M, N \rightarrow \infty$. Thus, $F_{N}(\theta)$ converges to $g \in\left[U \mathcal{M}_{q_{0}}^{p_{0}}, U \mathcal{M}_{q_{1}}^{p_{1}}\right]_{\theta}$. Hence, $\lim _{N \rightarrow \infty} F_{N}(\theta)=g$ in $\mathcal{M}_{q_{0}}^{p_{0}}+\mathcal{M}_{q_{1}}^{p_{1}}$. Meanwhile, by combining $\mathcal{M}_{q}^{p} \subseteq \mathcal{M}_{q_{0}}^{p_{0}}+\mathcal{M}_{q_{1}}^{p_{1}}$ and

$$
\lim _{N \rightarrow \infty}\left\|f-\chi_{\left\{\frac{1}{N} \leq|f| \leq N\right\}} f\right\|_{\mathcal{M}_{q}^{p}}=0,
$$

we have $\lim _{N \rightarrow \infty} F_{N}(\theta)=f$ in $\mathcal{M}_{q_{0}}^{p_{0}}+\mathcal{M}_{q_{1}}^{p_{1}}$, which implies $f=g$. Thus, $f \in$ $\left[U \mathcal{M}_{q_{0}}^{p_{0}}, U \mathcal{M}_{q_{1}}^{p_{1}}\right]_{\theta}$ as desired.

Theorem 5.7. Let $\theta \in(0,1), 1 \leq q_{0} \leq p_{0}<\infty, 1 \leq q_{1} \leq p_{1}<\infty$, and $\frac{p_{0}}{q_{0}}=\frac{p_{1}}{q_{1}}$. Define

Then we have

$$
\frac{1}{p}=\frac{1-\theta}{p_{0}}+\frac{\theta}{p_{1}} \quad \text { and } \quad \frac{1}{q}:=\frac{1-\theta}{q_{0}}+\frac{\theta}{q_{1}} .
$$

$$
\begin{aligned}
& {\left[U \mathcal{M}_{q_{0}}^{p_{0}}, U \mathcal{M}_{q_{1}}^{p_{1}}\right]^{\theta}} \\
& =\left\{f \in \mathcal{M}_{q}^{p}: \chi_{\{a \leq|f| \leq b\}} f \in U \mathcal{M}_{q}^{p} \text { for all } 0<a<b<\infty\right\} .
\end{aligned}
$$


From now on, we shall always use the assumption of Theorem 5.7. To prove Theorem 5.7, we shall invoke and prove several lemmas.

Lemma 5.8. [10] Keep the assumption in Theorem 5.7. Then

$$
U \bowtie \mathcal{M}_{q}^{p} \subseteq\left[U \mathcal{M}_{q_{0}}^{p_{0}}, U \mathcal{M}_{q_{1}}^{p_{1}}\right]^{\theta} .
$$

Proof. Without loss of generality, assume that $q_{0}>q_{1}$. Let $f \in U \bowtie \mathcal{M}_{q}^{p}$. Since $\chi_{\{a \leq|f| \leq b\}} \leq \frac{1}{a} \chi_{\{a \leq|f| \leq b\}}|f|$, we have $\chi_{\{a \leq|f| \leq b\}} \in U \mathcal{M}_{q}^{p}$. From Lemma 5.5, we have $\chi_{\{a \leq|f| \leq b\}} \in U \mathcal{M}_{q_{0}}^{p_{0}} \cap U \mathcal{M}_{q_{1}}^{p_{1}}$. For $z \in \bar{S}$, define

$$
F(z):=\operatorname{sgn}(f)|f|^{\frac{q z}{q_{0}}+\frac{q(1-z)}{q_{1}}} \text { and } G(z):=(z-\theta) \int_{0}^{1} F(\theta+(z-\theta) t) d t .
$$

Decompose $G(z)=G_{0}(z)+G_{1}(z)$ where $G_{0}(z):=\chi_{\{|f| \leq 1\}} G(z)$. Let $0<\varepsilon<1$. Since $\chi_{\{\varepsilon \leq|f| \leq 1\}} \in U \mathcal{M}_{q_{0}}^{p_{0}}$ and

$$
\chi_{\{\varepsilon \leq|f| \leq 1\}}\left|G_{0}(z)\right| \leq(1+|z|)\left(|f|^{q / q_{0}}+|f|^{q / q_{1}}\right) \chi_{\{\varepsilon \leq|f| \leq 1\}} \leq 2(1+|z|) \chi_{\{\varepsilon \leq|f| \leq 1\}},
$$

we have $\chi_{\{\varepsilon \leq|f| \leq 1\}} G_{0}(z) \in U \mathcal{M}_{q_{0}}^{p_{0}}$. Observe that

$$
\begin{aligned}
\left\|G_{0}(z)-\chi_{\{\varepsilon \leq|f| \leq 1\}} G_{0}(z)\right\|_{\mathcal{M}_{q_{0}}^{p_{0}}} & =\left\|\chi_{\{|f| \leq \varepsilon\}} \frac{F(z)-F(\theta)}{\left(\frac{q}{q_{1}}-\frac{q}{q_{0}}\right) \log |f|}\right\|_{\mathcal{M}_{q_{0}}^{p_{0}}} \\
& \leq\left\|\frac{2|f|^{q / q_{0}}}{\left(\frac{q}{q_{1}}-\frac{q}{q_{0}}\right) \log \left(\varepsilon^{-1}\right)}\right\|_{\mathcal{M}_{q_{0}}^{p_{0}}} \\
& =\frac{2\|f\|_{\mathcal{M}_{q}^{p}}^{q / q_{0}}}{\left(\frac{q}{q_{1}}-\frac{q}{q_{0}}\right) \log \varepsilon^{-1}} \rightarrow 0
\end{aligned}
$$

as $\varepsilon \rightarrow 0^{+}$. Hence $G_{0}(z) \in U \mathcal{M}_{q_{0}}^{p_{0}}$. Similarly, $G_{1}(z) \in U \mathcal{M}_{q_{1}}^{p_{1}}$. Thus $G(z) \in$ $U \mathcal{M}_{q_{0}}^{p_{0}}+U \mathcal{M}_{q_{1}}^{p_{1}}$. Let $t \in \mathbb{R}$ and $R>1$. Since $\chi_{\left\{R^{-1} \leq|f| \leq R\right\}} \in U \mathcal{M}_{q_{0}}^{p_{0}}$ and

$$
|(G(i t)-G(0))| \chi_{\left\{R^{-1} \leq|f| \leq R\right\}} \leq(2+|t|)\left(R^{q / q_{0}}+R^{q / q_{1}}\right) \chi_{\left\{R^{-1} \leq|f| \leq R\right\}},
$$

we have $[G(i t)-G(0)] \chi_{\left\{R^{-1} \leq|f| \leq R\right\}} \in U \mathcal{M}_{q_{0}}^{p_{0}}$. Note that

$$
\left\|[G(i t)-G(0)] \chi_{\mathbb{R}^{n} \backslash\left\{R^{-1} \leq|f| \leq R\right\}}\right\|_{\mathcal{M}_{q_{0}}^{p_{0}}} \leq \frac{2\|f\|_{\mathcal{M}_{q}^{p}}^{q / q_{0}}}{\left(\frac{q}{q_{1}}-\frac{q}{q_{0}}\right) \log R} \rightarrow 0
$$

as $R \rightarrow \infty$. Thus $G(i t)-G(0) \in U \mathcal{M}_{q_{0}}^{p_{0}}$. Similarly, $G(1+i t)-G(1) \in U \mathcal{M}_{q_{1}}^{p_{1}}$. Since $G \in \mathcal{G}\left(\mathcal{M}_{q_{0}}^{p_{0}}, \mathcal{M}_{q_{1}}^{p_{1}}\right)$, we have $G \in \mathcal{G}\left(U \mathcal{M}_{q_{0}}^{p_{0}}, U \mathcal{M}_{q_{1}}^{p_{1}}\right)$. From $f=G^{\prime}(\theta)$, it follows that $f \in\left[U \mathcal{M}_{q_{0}}^{p_{0}}, U \mathcal{M}_{q_{1}}^{p_{1}}\right]^{\theta}$.

Lemma 5.9. [10] Let $G \in \mathcal{G}\left(\mathcal{M}_{q_{0}}^{p_{0}}, \mathcal{M}_{q_{1}}^{p_{1}}\right)$ and $\theta \in(0,1)$. For $z \in \bar{S}$ and $k \in \mathbb{N}$, define $H_{k}(z)$ by $(22)$. Then $H_{k}(\theta) \in \overline{U \mathcal{M}_{q_{0}}^{p_{0}} \cap U \mathcal{M}_{q_{1}}^{p_{1}}} \mathcal{M}_{q}^{p}$. 
Proof. It follows from Lemma 3.11, that $H_{k}(\theta) \in\left[U \mathcal{M}_{q_{0}}^{p_{0}}, U \mathcal{M}_{q_{1}}^{p_{1}}\right]_{\theta}$. Let $\varepsilon>0$. Since $U \mathcal{M}_{q_{0}}^{p_{0}} \cap U \mathcal{M}_{q_{1}}^{p_{1}}$ is dense in $\left[U \mathcal{M}_{q_{0}}^{p_{0}}, U \mathcal{M}_{q_{1}}^{p_{1}}\right]_{\theta}$, we can find $J_{k}(\theta) \in U \mathcal{M}_{q_{0}}^{p_{0}} \cap U \mathcal{M}_{q_{1}}^{p_{1}}$ such that

$$
\left\|H_{k}(\theta)-J_{k}(\theta)\right\|_{\left[U \mathcal{M}_{q_{0}}^{p_{0}}, U \mathcal{M}_{q_{1}}^{p_{1}}\right]_{\theta}}<\varepsilon .
$$

Since $\left[U \mathcal{M}_{q_{0}}^{p_{0}}, U \mathcal{M}_{q_{1}}^{p_{1}}\right]_{\theta} \subseteq\left[\mathcal{M}_{q_{0}}^{p_{0}}, \mathcal{M}_{q_{1}}^{p_{1}}\right]_{\theta} \subseteq \mathcal{M}_{q}^{p}$, we have

$$
\left\|H_{k}(\theta)-J_{k}(\theta)\right\|_{\mathcal{M}_{q}^{p}} \lesssim\left\|H_{k}(\theta)-J_{k}(\theta)\right\|_{\left[U \mathcal{M}_{q_{0}}^{p_{0}}, U \mathcal{M}_{q_{1}}^{p_{1}}\right]_{\theta}}<\varepsilon .
$$

This shows that $H_{k}(\theta) \in \overline{U \mathcal{M}_{q_{0}}^{p_{0}} \cap U \mathcal{M}_{q_{1}}^{p_{1}}} \mathcal{M}_{q}^{p}$.

Lemma 5.10. [10] Under the assumption of Theorem 5.7, we have

$$
\mathcal{M}_{q}^{p} \cap{\overline{U \mathcal{M}_{q}^{p}}}^{\mathcal{M}_{q_{0}}^{p_{0}}+\mathcal{M}_{q_{1}}^{p_{1}}} \subseteq U \bowtie \mathcal{M}_{q}^{p} .
$$

Proof. Let $f \in \mathcal{M}_{q}^{p} \cap{\overline{U \mathcal{M}_{q}^{p}}}^{\mathcal{M}_{q_{0}}^{p_{0}}+\mathcal{M}_{q_{1}}^{p_{1}}}$. Choose $\left\{f_{j}\right\}_{j=1}^{\infty} \subseteq U \mathcal{M}_{q}^{p}$ such that

$$
\lim _{j \rightarrow \infty}\left\|f-f_{j}\right\|_{\mathcal{M}_{q_{0}}^{p_{0}}+\mathcal{M}_{q_{1}}^{p_{1}}}=0 .
$$

Then, we can find $\left\{k_{j}\right\}_{j=1}^{\infty} \subset U \mathcal{M}_{q_{0}}^{p_{0}}$ and $\left\{h_{j}\right\}_{j=1}^{\infty} \subset U \mathcal{M}_{q_{1}}^{p_{1}}$ convergent to 0 in $U \mathcal{M}_{q_{0}}^{p_{0}}$ and $U \mathcal{M}_{q_{1}}^{p_{1}}$, respectively, such that $f-f_{j}=k_{j}+h_{j}$ for all $j$. Assume $0<a<1<b<\infty$ as before. Let $\Theta \in C_{\mathrm{c}}(\mathbb{R})$ be a piecewise linear function defined by

$$
\Theta^{\prime}(t):=\frac{2}{a} \chi_{(a / 2, a)}(t)-\frac{1}{b} \chi_{(b, 2 b)}(t)
$$

except at $t=\frac{a}{2}, a, b, 2 b$. Let $C_{a, b}=\frac{2}{a}+\frac{1}{b}$. Since

$$
|\Theta(t)-\Theta(s)| \leq C_{a, b}|t-s| \text { and }|\Theta(t)-\Theta(s)| \leq 2,
$$

we have

$$
\left|\Theta(|f|)-\Theta\left(\left|f_{j}\right|\right)\right| \leq C_{a, b} \min \left(1,|| f|-| f_{j}||\right) \leq C_{a, b} \min \left(1,\left|f-f_{j}\right|\right) .
$$

Let $B=B\left(x_{0}, r\right)$ be any ball in $\mathbb{R}^{n}$. Then,

$$
\begin{aligned}
\int_{B} \chi_{[a, b]}(|f(x)|) \mid & \Theta(|f(x)|)-\left.\Theta\left(\left|f_{j}(x)\right|\right)\right|^{q} d x \\
& \lesssim \int_{B} \chi_{[a, b]}(|f(x)|) \min \left(1,\left|f(x)-f_{j}(x)\right|^{q}\right) d x .
\end{aligned}
$$

By using the decomposition $f=f_{j}+k_{j}+h_{j}$, we obtain

$$
\begin{aligned}
& \int_{B} \chi_{[a, b]}(|f(x)|)\left|\Theta(|f(x)|)-\Theta\left(\left|f_{j}(x)\right|\right)\right|^{q} d x \\
& \lesssim \int_{B} \chi_{[a, b]}(|f(x)|) \min \left(1,\left|k_{j}(x)\right|^{q}\right) d x+\int_{B} \chi_{[a, b]}(|f(x)|) \min \left(1,\left|h_{j}(x)\right|^{q}\right) d x .
\end{aligned}
$$


Keeping in mind, $q_{0}>q>q_{1}$ and $\frac{q_{0}}{p_{0}}=\frac{q_{1}}{p_{1}}=\frac{q}{p}$. Then

$$
\begin{aligned}
& |B|^{\frac{q}{p}-1} \int_{B} \chi_{[a, b]}(|f(x)|)\left|\Theta(|f(x)|)-\Theta\left(\left|f_{j}(x)\right|\right)\right|^{q} d x \\
& \lesssim|B|^{\frac{q}{p}-1} \int_{B}\left|h_{j}(x)\right|^{q_{1}} d x \\
& \quad+|B|^{\frac{q}{p}-1}\left(\int_{B} \chi_{[a, b]}(|f(x)|) d x\right)^{1-\frac{q}{q_{0}}}\left(\int_{B} \min \left(1,\left|k_{j}(x)\right|^{q_{0}}\right) d x\right)^{\frac{q}{q_{0}}} \\
& \lesssim\left(\left\|h_{j}\right\|_{\mathcal{M}_{q_{1}}^{p_{1}}}\right)^{q_{1}}+\left(|B|^{\frac{q}{p}-1} \int_{B}|f(x)|^{q} d x\right)^{1-\frac{q}{q_{0}}}\left(\left\|k_{j}\right\|_{\mathcal{M}_{q_{0}}^{p_{0}}}\right)^{q} \\
& \lesssim\left(\left\|h_{j}\right\|_{\mathcal{M}_{q_{1}}^{p_{1}}}\right)^{q_{1}}+\left(|B|^{\frac{q}{p}-1} \int_{B}|f(x)|^{q} d x\right)^{1-\frac{q}{q_{0}}}\left(\left\|k_{j}\right\|_{\mathcal{M}_{q_{0}}^{p_{0}}}\right)^{q} \\
& \lesssim\left(\left\|h_{j}\right\|_{\mathcal{M}_{q_{1}}^{p_{1}}}\right)^{q_{1}}+\left(\|f\|_{\mathcal{M}_{q}^{p}}\right)^{q-\frac{q^{2}}{q_{0}}}\left(\left\|k_{j}\right\|_{\mathcal{M}_{q_{0}}^{p_{0}}}\right)^{q} .
\end{aligned}
$$

Thus, it follows that

$$
\lim _{j \rightarrow \infty}\left\|\chi_{\{a \leq|f| \leq b\}} \Theta\left(\left|f_{j}\right|\right)-\chi_{\{a \leq|f| \leq b\}} \Theta(|f|)\right\|_{\mathcal{M}_{q}^{p}}=0 .
$$

Since $\chi_{\{a \leq|f| \leq b\}} \Theta\left(\left|f_{j}\right|\right) \leq a^{-1}\left|f_{j}\right|$, we have $\chi_{\{a \leq|f| \leq b\}} \Theta(|f|) \in U \mathcal{M}_{q}^{p}$. From the equality

it follows that $\chi_{\{a \leq|f| \leq b\}} f \in U \mathcal{M}_{q}^{p}$.

$$
\chi_{\{a \leq|f| \leq b\}}|f|=b \chi_{\{a \leq|f| \leq b\}} \Theta(|f|),
$$

Now, we are ready to prove Theorem 5.7.

Theorem 5.7. In view of Lemma 5.8, we only need to show that

$$
\left[U \mathcal{M}_{q_{0}}^{p_{0}}, U \mathcal{M}_{q_{1}}^{p_{1}}\right]^{\theta} \subseteq U \bowtie \mathcal{M}_{q}^{p} .
$$

Let $f \in\left[U \mathcal{M}_{q_{0}}^{p_{0}}, U \mathcal{M}_{q_{1}}^{p_{1}}\right]^{\theta}$. Then there exists $G \in \mathcal{G}\left(U \mathcal{M}_{q_{0}}^{p_{0}}, U \mathcal{M}_{q_{1}}^{p_{1}}\right)$ such that $G^{\prime}(\theta)=f$. For $z \in \bar{S}$ and $k \in \mathbb{N}$, define $H_{k}(z)$ by (22). By virtue of Lemmas 5.6 and 5.9, we have $H_{k}(\theta) \in U \mathcal{M}_{q}^{p}$. Since $H_{k}(\theta)$ converges to $G^{\prime}(\theta)=f$ in $\mathcal{M}_{q_{0}}^{p_{0}}+\mathcal{M}_{q_{1}}^{p_{1}}$, we see that $f \in U \bowtie \mathcal{M}_{q}^{p}$.

By substituting $U:=L_{\mathrm{c}}^{\infty}, L_{\mathrm{c}}^{0}, L^{\infty}$, we have the following result.

Corollary 5.11. Keep the same assumption as in the previous theorems. Then

$$
\begin{aligned}
{\left[\widetilde{\mathcal{M}}_{q_{0}}^{p_{0}}, \widetilde{\mathcal{M}}_{q_{1}}^{p_{1}}\right]_{\theta}=\left[\mathcal{M}_{q_{0}}^{p_{0}}, \mathcal{M}_{q_{1}}^{p_{1}}\right]_{\theta}=\widetilde{\mathcal{M}}_{q}^{p}, } & \\
{\left[\overline{\mathcal{M}}_{q_{0}}^{p_{0}}, \overline{\mathcal{M}}_{q_{1}}^{p_{1}}\right]_{\theta} } & =\left\{f \in \overline{\mathcal{M}}_{q}^{p}: \lim _{N \rightarrow \infty}\left\|f \chi_{\left\{|f|<\frac{1}{N}\right\}}\right\|_{\mathcal{M}_{q}^{p}}=0\right\}, \\
{\left[\widetilde{\mathcal{M}}_{q_{0}}^{p_{0}}, \widetilde{\mathcal{M}}_{q_{1}}^{p_{1}}\right]^{\theta} } & =\left[\mathcal{M}_{q_{0}}^{p_{0}}, \mathcal{\mathcal { M }}_{q_{1}}^{p_{1}}\right]^{\theta} \\
& =\bigcap_{0<a<b<\infty}\left\{f \in \mathcal{M}_{q}^{p}: \chi_{\{a \leq|f| \leq b\}} f \in \widetilde{\mathcal{M}}_{q}^{p}\right\},
\end{aligned}
$$


and $\left[\overline{\mathcal{M}}_{q_{0}}^{p_{0}}, \overline{\mathcal{M}}_{q_{1}}^{p_{1}}\right]^{\theta}=\mathcal{M}_{q}^{p}$

\section{ACKNOWLEDGEMENT}

The author is thankful to Professor Yoshihiro Sawano for his helpful assistance with this note.

\section{REFERENCES}

[1] J. Bergh and J. Löfström, Interpolation spaces. An introduction, Grundlehren der Mathematischen Wissenschaften, no. 223. Springer-Verlag, Berlin-New York, 1976.

[2] J. Bergh, On the relation between the two complex methods of interpolation. Indiana Univ. Math. J. 28(5), (1979), 775-778.

[3] O. Blasco, A. Ruiz and L. Vega, Non-interpolation in Morrey-Campanato and block spaces, Ann. Scuola Norm. Sup. Pisa Cl. Sci., 28 (1999), 31-40.

[4] A.P. Calderón, Intermediate spaces and interpolation, the complex method, Studia Math. 24 (1964), no.2, 113-190.

[5] L. Caso, R. D'Ambrosio, and S. Monsurrò, Some remarks on spaces of Morrey type. Abstr. Appl. Anal., Art. ID 242079, 22 pp. ( 2010).

[6] F. Cobos, J. Peetre and L. E. Persson, On the connection between real and complex interpolation of quasi-Banach spaces, Bull. Sci. Math. 122 (1998), 17-37.

[7] L. Grafakos, Classical Fourier Analysis, Second Edition, Springer, 2008.

[8] J. Hadamard, Sur les fonctions entières, Bull. Soc. Math. France 24, (1896), 186-187.

[9] D.I. Hakim and Y. Sawano, Interpolation of generalized Morrey spaces, Rev. Mat. Complut. 29 (2016), 295-340.

[10] D.I. Hakim and Y. Sawano, Calderón's first and second complex interpolations of closed subspaces of Morrey spaces, J. Fourier Anal. Appl. 23 (2017), no. 5, 1195-1226.

[11] D. I. Hakim, Complex interpolation of certain closed subspaces of Morrey spaces, Tokyo J. Math. 41 (2018), no. 2, 487-514.

[12] D.I. Hakim, Complex interpolation of generalized Morrey spaces, local block spaces, and grand Lebesgue spaces, Dissertation, Tokyo Metropolitan University (2018).

[13] D.I. Hakim, S. Nakamura, and Y. Sawano, Complex interpolation of smoothness Morrey subspaces, Constr Approx. 46, (2017), 489-563.

[14] P. G. Lemarié-Rieusset, Erratum to: Multipliers and Morrey spaces, Potential Anal. 41 (2014), no.4, 1359-1362.

[15] Y. Lu, D. Yang, and W. Yuan, Interpolation of Morrey spaces on metric measure spaces, Canad. Math. Bull. 57 (2014), 598-608.

[16] C.B. Morrey, On the solutions of quasi linear elliptic partial differential equations, Trans. Amer. Math. Soc. 43 (1938), 126-166.

[17] A. Ruiz and L. Vega, Corrigenda to "Unique continuation for Schrödinger operators with potential in Morrey spaces" and a remark on interpolation of Morrey spaces, Publ. Mat. 39 (1995), 405-411.

[18] V.A. Sestakov, On complex interpolation of Banach space of measurable functions, Vestnik Leningrad, 19 (4), (1974), 64-68.

[19] G. Stampacchia, The spaces $\mathcal{L}^{(p, \lambda)}, N^{(p, \lambda)}$ and interpolation. Ann. Scuola Norm. Sup. Pisa 19 (1965), 443-462.

[20] D. Yang, W. Yuan, and C. Zhuo, Complex interpolation on Besov-Type and Triebel-LizorkinType Spaces, Anal. and Appl., (2013), 45pp.

[21] W. Yuan, W. Sickel and D. Yang, Interpolation of Morrey-Campanato and related smoothness spaces, Sci. China Math. 58, no. 9, (2015), 1835-1908. 